\title{
Valeur alimentaire pour les ruminants des légumineuses contenant des tannins condensés en milieux tempérés
}

\author{
J. AUFRERE ${ }^{1,2}$, K. THEODORIDOU ${ }^{1,2,3}$, R. BAUMONT 1,2 \\ ${ }^{1}$ INRA, UMR1213 Herbivores, F-63122 Saint-Genès-Champanelle, France \\ ${ }^{2}$ Clermont Université, VetAgro Sup, UMR Herbivores, BP 10448, F-63000, Clermont-Ferrand, France \\ ${ }^{3}$ University of Saskatchewan, Saskatoon, SK S S7N 5A8, Canada \\ Courriel : jocelyne.aufrere@clermont.inra.fr
}

Les légumineuses fourragères contenant des tannins condensés ont des propriétés intéressantes pour la nutrition et la santé des ruminants et offrent des perspectives pour limiter les rejets animaux. Cela explique le regain d'intérêt pour ces plantes dont la valeur alimentaire a été très peu étudiée.

Aujourd'hui, l'agriculture doit produire différemment en respectant davantage l'environnement. Les récentes crises alimentaires mondiales, conduisent à une augmentation du prix des intrants qui fragilise les élevages et incite à utiliser plus de fourrages dans l'alimentation des ruminants. Maximiser la valeur alimentaire des fourrages est un élément clé pour limiter le recours aux aliments complémentaires dans l'alimentation des ruminants et ainsi accroître l'autonomie alimentaire des systèmes d'élevages.

Les légumineuses fourragères présentent beaucoup d'atouts notamment par leur teneur élevée en azote et par leur capacité à fixer l'azote atmosphérique qui dispense de la nécessité d'apporter une fertilisation azotée. La solubilité des protéines foliaires est en revanche très élevée pouvant conduire à une dégradation importante de ces protéines dans le rumen jusqu'au stade ammoniac et donc à une proportion importante d'azote alimentaire perdue sous forme urinaire. Les Tannins Condensés (TC) accumulés dans les vacuoles de certaines légumineuses peuvent limiter cette dégradation. Lors de la mastication, ils se lient aux protéines alimentaires ou microbiennes et forment des complexes peu solubles et non dégradés dans le rumen. Présents également dans les dicotylédones, dans certains arbres, arbustes et fourrages tropicaux, ces métabolites ont la fonction de protéger les plantes dans des conditions de stress et de maintenir leur intégrité contre les compétiteurs, les prédateurs et les agents pathogènes, et de conserver leur énergie et leurs matières azotées pour des mobilisations futures (Waghorn 2008). Suivant leur teneur dans la plante et leur structure, les TC vont avoir un effet bénéfique ou délétère sur la valeur alimentaire du fourrage et sur les performances animales. Alors que de nombreux travaux ont été réalisés en Nouvelle Zélande, peu de travaux ont porté sur la valeur alimentaire des fourrages contenant des TC en zone tempérée en France, et en Europe.

Dans ce texte nous ferons le point sur la valeur alimentaire des légumineuses contenant des TC, tout en soulignant leur intérêt pour limiter les pertes d'azote lessivable et les pertes de méthane voire de composés azotés gazeux dans l'environnement. Les effets des TC sur la santé animale (absence de météorisation, propriétés antiparasitaires) ne seront pas abordés. Une partie importante des résultats présentés ici concerne le sainfoin en raison d'expérimentations récentes effectuées dans le cadre d'un programme européen «Healthyhay» entre 2007 et 2010 (Theodoridou 2010, Theodoridou et al 2010, 2011a, b, 2012). La comparaison avec la valeur alimentaire d'autres fourrages contenant des TC (lotier, sulla...) ou non (luzerne, trèfle) se limitera aux fourrages des zones tempérées. La plupart des expériences in vivo rapportées dans ce texte ont été réalisées chez le mouton. Lorsqu'une autre espèce animale a été utilisée, cette dernière est signalée dans le texte.

\section{1 / Les tannins condensés des légumineuses des mi- lieux tempérés}

Les TC sont des composés secondaires présents dans les vacuoles des cellules des feuilles, des tiges, des racines, des fleurs et des enveloppes des graines. Dans les zones tempérées, ce sont principalement ceux des tiges et des feuilles qui jouent un rôle dans la nutrition du ruminant. Ils ne participent pas directement au développement de la plante. Ils ont un rôle de protection contre les rayons ultra-violets et la sécheresse. D'après Waghorn (2008), leur rôle écologique est de protéger les plantes contre des prédateurs naturels (insectes, herbivores), or ils sont assez inefficaces dans ce rôle puisque les chèvres et les moutons sélectionnent de préférence les feuilles plus riches en TC que les tiges.

\section{1 / Structure biochimique}

Les TC sont synthétisés dans le cytoplasme des cellules à partir de la phenylalanine et de l'acétate pour former des unités de catéchine dans les vacuoles des cellules. Ce sont des polymères de flavanoïdes (flavan-3ols) liés par des liaisons C-C. Les monomères peuvent être liés par des liaisons C4-C8 ou C4-C6 qui affectent la forme de la chaîne de polymères. Différentes combinaisons des groupes $\mathrm{OH}$ et $\mathrm{H}$ des unités de monomères conduisent à différentes classes de 
Figure 1. Structure des Tannins Condensés (TC). Au niveau de chacun des n monomères, si $R=H$, la structure représente la ProCyanidine $(P C)$, si $R=O H$, la structure représente la ProDelphinidine (PD).

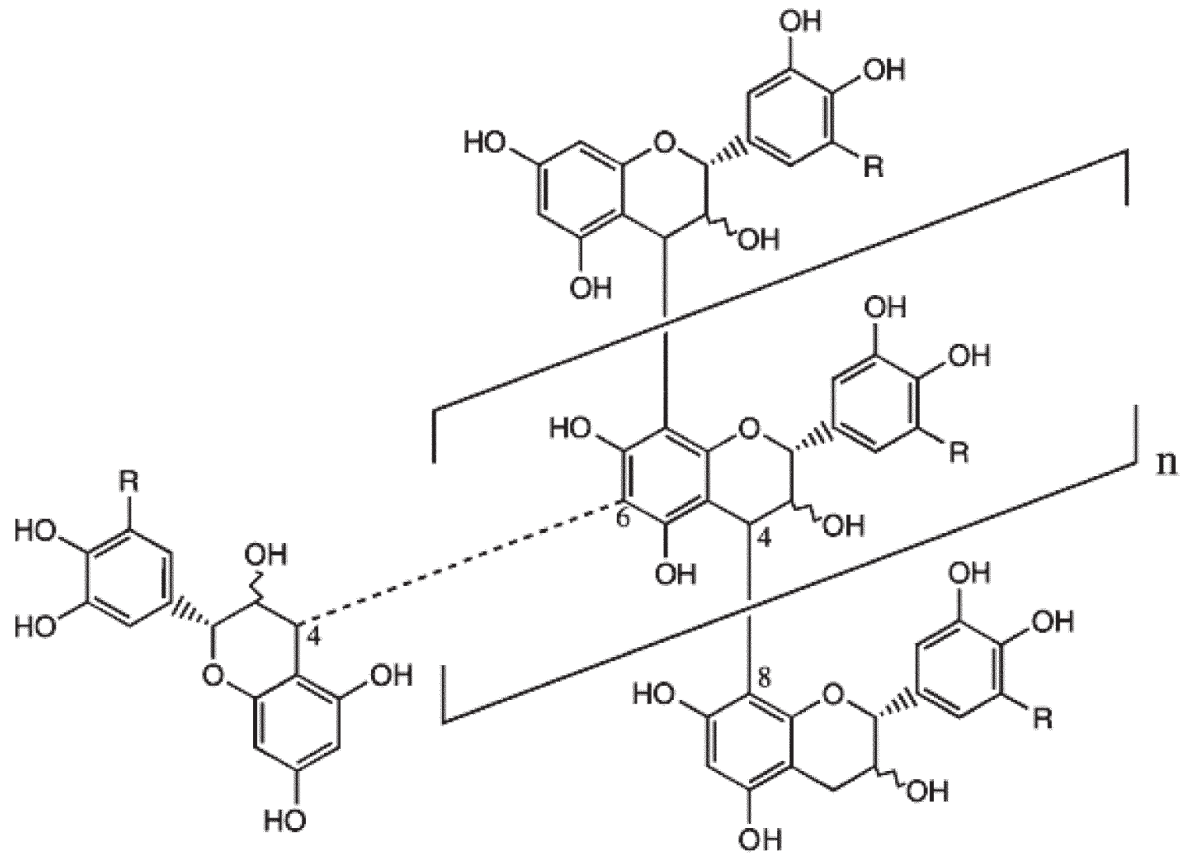

polymères : ProDelphynidines (PD) et ProCyanidines (PC) (figure 1).

Les effets des tannins vont être modulés par différents facteurs tels que leur teneur dans la plante et les paramètres de leur structure : le Degré moyen de Polymérisation (mDP) (i.e. poids moléculaire moyen), le rapport prodelphinidine/procyanidine (PD/PC), la conformation dans l'espace (forme cis ou trans), la capacité des tannins à se lier aux protéines et le rapport tannins/protéines (Spencer et al 1988, Silber et al 1998, McAllister et al 2005, Frazier et al 2010).

A l'exception de travaux sur le lotier corniculé et pédonculé, peu d'études existent sur la structure des TC des fourrages. De plus, les effets des paramètres de la structure sont complexes à interpréter et peuvent agir en sens opposé. Ainsi, lorsque que le rapport PD/PC augmente, la capacité des tannins à se lier aux protéines doit augmenter du fait de la prédominance des groupes $\mathrm{OH}$ capables de se fixer aux protéines. C'est ce que l'on observe pour le lotier pédonculé, dont le rapport $\mathrm{PD} / \mathrm{PC}$ est plus élevé que celui du lotier corniculé. En général, les effets bénéfiques ou délétères des TC du lotier corniculé ou pédonculé sur les performances animales sont attribués à la prédominance de $\mathrm{PC}$ ou PD chez cette espèce (Waghorn 2008). Mais, le rapport PD/PC des TC du sainfoin (Theodoridou et al 2010) et du sulla (Tibe et al 2011) est comparable à celui des TC du lotier pédonculé, alors

En raison de la grande variation de
Unité terminale

qui est à la fois qualitative et quantitative, permet de mesurer la teneur en TC et d'avoir des indications sur leur structure. Cependant à l'heure actuelle, elle n'est pas applicable à tous les modes de conservation du fourrage, en particulier aux ensilages.

Ainsi, le concept selon lequel une teneur en TC dans la plante inférieure à $50 \mathrm{~g} / \mathrm{kg}$ MS serait bénéfique pour la santé et la nutrition de l'animal (Barry et Mc Naab 1999) est obsolète et ne reste valable que lorsque le dosage a été réalisé par la méthode vaniline- $\mathrm{HCl}$ ou par la méthode $\mathrm{HCl}$-butanol. De plus, les recommandations de Barry et McNaab (1999) proviennent d'expérimentations réalisées avec du lotier et ne sont pas applicables pour d'autres fourrages contenant une concentration plus élevée en TC estimée par la même méthode d'analyse. Par exemple le sulla (teneur en $\mathrm{TC}=72 \mathrm{~g} / \mathrm{kg} \mathrm{MS}$ ) et le sainfoin (teneur en $\mathrm{TC}=80 \mathrm{~g} / \mathrm{kg} \mathrm{MS}$ ) ont une valeur nutritive élevée (Ulyatt et Egan 1979, Stienezen et al 1996). La teneur plus faible en TC du lotier corniculé par rapport à celle du lotier pédonculé n'est pas suffisante à elle seule pour expliquer leurs effets différents du point de vue nutritionnel.

que les effets de ces fourrages sur paramètres digestifs sont différents de ceux du lotier pédonculé. Des différences de poids moléculaire des TC entre lotier corniculé $(5300 \mathrm{Da})$ et pédonculé (12300Da) (Meagher et al 2004) pourraient expliquer des effets différents sur les paramètres de la valeur alimentaire (ingestibilité, digestibilité) entre ces deux types de plantes. Cela laisse penser que d'autres paramètres peuvent moduler l'effet des TC (Mueller-Harvey 2006).

\section{2 / Teneur en tannins conden- sés et méthodes de dosage} leur structure, la mesure de la teneur en TC est complexe. Les méthodes de dosage les plus couramment utilisées sont des méthodes colorimétriques (voir encadré) : HCL-butanol (Porter et al 1986, Terrill et al 1992a), méthode à la vanilline, (Broadhurst et Jones 1978, Terrill et al 1992a) ou basées sur la précipitation des protéines (méthode de diffusion radiale, Hagerman 1987). Ces méthodes permettent de doser des entités différentes et restent imparfaites en raison notamment d'un manque de standards appropriés et de leur non spécificité. La méthode de diffusion radiale permet de déterminer l'activité biologique mais est mal adaptée pour mesurer la teneur en TC. La comparaison des résultats des différents travaux de la littérature reste donc difficile (Schofield et al 2001). Enfin, très récemment, la méthode par la thiolyse (Gea et al 2011)

\section{3 / Activité biologique ou capa- cité des tannins condensés à se lier aux protéines}

L'activité biologique, ou astringence, représente la capacité des TC à se lier aux protéines, mais ce sont seulement les tannins extractibles qui vont réagir avec les protéines. Chez le ruminant, les TC d'un fourrage vont se lier avec la rubisco, principale protéine des fourrages verts ou avec des protéines de la salive, des protéines microbiennes, des enzymes et des protéines endogènes. L'activité biologique peut être mesurée au laboratoire mais cette méthode comme les méthodes colorimétriques nécessite l'utilisation d'un standard pour sa quantification. Ce dernier est l'acide tannique, et l'activité biologique est exprimée en $g$ équivalent acide tannique/100 g MS. Les études de Mc Allister et al (2005) réalisées avec 9 fourrages d'espèces différentes contenant des TC, indiquent que la capacité des TC à se lier à la rubisco est plus élevée que leur capacité à se lier à l'albumine de sérum bovin utilisée comme standard dans la méthode de diffusion radiale. Les TC se lieraient de façon très efficace aux unités de rubisco de poids moléculaire élevé et plus faiblement aux unités de poids moléculaire plus faible (Min et al 2003). La teneur et la structure chimique des TC apparaissent comme les principaux facteurs qui expliquent les variations de leur capacité à se lier aux protéines pour des $\mathrm{pH}$ 


\begin{tabular}{|c|c|c|c|c|}
\hline Méthode & Type et principe & Avantages & Inconvénients & Remarques \\
\hline $\begin{array}{l}\text { HCL-butanol } \\
\text { Porter et al (1986), } \\
\text { Terrill et al (1992a) }\end{array}$ & $\begin{array}{l}\text { Méthode chimique. } \\
\text { Dosage } \\
\text { colorimétrique. } \\
\text { Dépolymérisation } \\
\text { oxydative en milieu } \\
\text { acide des TC } \\
\text { conduisant à la } \\
\text { production } \\
\text { d'anthocyanes de } \\
\text { couleur rouge. }\end{array}$ & $\begin{array}{l}\text { Spécifique des } \\
\text { TC. } \\
\text { Simple, rapide. }\end{array}$ & $\begin{array}{l}\text { Nécessite des standards } \\
\text { externes. } \\
\text { L'intensité de la coloration } \\
\text { varie avec la structure des } \\
\text { TC. } \\
\text { Les polymères des TC sont } \\
\text { clivés en dimères et } \\
\text { trimères plus qu'en } \\
\text { monomères ce qui conduit } \\
\text { à une sous-estimation. }\end{array}$ & $\begin{array}{l}\text { Méthode mesurant le } \\
\text { nombre total de } \\
\text { résidus de } \\
\text { flavonoïdes. }\end{array}$ \\
\hline $\begin{array}{l}\text { Vanilline } \\
\text { Broadhurst et Jones } \\
\text { (1978), } \\
\text { Terrill et al (1992a) }\end{array}$ & $\begin{array}{l}\text { Méthode chimique. } \\
\text { Dosage } \\
\text { colorimétrique. } \\
\text { La vanilline (aldéhyde } \\
\text { aromatique) réagit } \\
\text { avec les TC pour } \\
\text { former un complexe } \\
\text { coloré. }\end{array}$ & & $\begin{array}{l}\text { La structure des TC affecte } \\
\text { l'intensité de la coloration. } \\
\text { Manque de spécificité. }\end{array}$ & $\begin{array}{l}\text { Méthode mesurant } \\
\text { les flavonoïdes et les } \\
\text { tannins totaux. } \\
\text { Inappropriée pour la } \\
\text { détermination des TC } \\
\text { liés aux parois } \\
\text { végétales. }\end{array}$ \\
\hline $\begin{array}{l}\text { Folin et Ciocalteu } \\
\text { Folin et Denis (1912) }\end{array}$ & $\begin{array}{c}\text { Méthode } \\
\text { colorimétrique. } \\
\text { Mesure de la tyrosine } \\
\text { des composés } \\
\text { phénoliques. }\end{array}$ & $\begin{array}{l}\text { Dosage des } \\
\text { polyphénols } \\
\text { totaux. }\end{array}$ & $\begin{array}{l}\text { Formation de précipités qui } \\
\text { interfèrent avec les } \\
\text { mesures } \\
\text { spectrophotométriques. }\end{array}$ & $\begin{array}{l}\text { Méthode non } \\
\text { spécifique. }\end{array}$ \\
\hline $\begin{array}{l}\text { Thiolyse } \\
\text { Gea et al (2011) }\end{array}$ & $\begin{array}{l}\text { Méthode chimique. } \\
\text { Utilisation de HPLC. }\end{array}$ & $\begin{array}{c}\text { Permet la } \\
\text { détermination de } \\
\text { la teneur en TC } \\
\text { et de leur } \\
\text { structure. } \\
\end{array}$ & $\begin{array}{c}\text { Nécessité d'avoir des TC } \\
\text { purs. } \\
\text { Problème avec du matériel } \\
\text { hétérogène (prairies, } \\
\text { ensilages). }\end{array}$ & $\begin{array}{c}\text { Méthode d'avenir } \\
\text { pour la détermination } \\
\text { de la teneur et } \\
\text { structure des TC } \\
\text { dans les fourrages. }\end{array}$ \\
\hline $\begin{array}{l}\text { Gravimétrique } \\
\text { Reed et al (1985) }\end{array}$ & $\begin{array}{l}\text { Mesure les TC liés au } \\
\text { polyvinyl pyrrolidone } \\
\text { ou précipitation des } \\
\text { polyphénols solubles } \\
\text { avec l'ytterbium. }\end{array}$ & $\begin{array}{l}\text { Utilisation de } \\
\text { standard non } \\
\text { nécessaire. }\end{array}$ & $\begin{array}{l}\text { Moins sensible que } \\
\text { méthodes colorimétriques. } \\
\text { Rendement de la réaction } \\
\text { variable avec le rapport } \\
\text { Ytterbium } / \text { annin. } \\
\text { Mesures faites sur les } \\
\text { matières minérales. }\end{array}$ & $\begin{array}{l}\text { Utilisable pour } \\
\text { préparer des } \\
\text { standards pour } \\
\text { méthodes } \\
\text { colorimétriques. }\end{array}$ \\
\hline $\begin{array}{l}\text { Activité } \\
\text { enzymatique } \\
\text { Goldstein et Swain } \\
\text { (1965) }\end{array}$ & $\begin{array}{c}\text { Inhibition de l'activité } \\
\text { de certaines enzymes } \\
\text { par les tannins. }\end{array}$ & $\begin{array}{c}\text { Haute sensibilité } \\
\text { parce que les } \\
\text { enzymes ont un } \\
\text { rôle } \\
\text { amplificateur. }\end{array}$ & $\begin{array}{c}\text { Certaines enzymes sont } \\
\text { plus adaptées que } \\
\text { d'autres. }\end{array}$ & $\begin{array}{l}\text { Ne peut pas être } \\
\text { mise en relation avec } \\
\text { l'activité biologique. }\end{array}$ \\
\hline $\begin{array}{l}\text { Diffusion Radiale } \\
\text { Hagerman (1987) }\end{array}$ & $\begin{array}{c}\text { Mesure de l'activité } \\
\text { biologique. } \\
\text { Formation de } \\
\text { complexes entre les } \\
\text { tannins de la plante et } \\
\text { l'albumine sérique } \\
\text { bovine inclus dans } \\
\text { une solution d'agar. }\end{array}$ & $\begin{array}{l}\text { Méthode rapide. } \\
\text { Ne requiert ni } \\
\text { réactifs } \\
\text { complexes, ni } \\
\text { instrumentation. }\end{array}$ & $\begin{array}{l}\text { Moins précise que les } \\
\text { méthodes colorimétriques } \\
\text { pour la quantification. } \\
\text { Les tannins condensés et } \\
\text { hydrolysables peuvent être } \\
\text { détectés. } \\
\text { Le résultat dépend du } \\
\text { choix de la protéine. }\end{array}$ & $\begin{array}{l}\text { Mesure de l'activité } \\
\text { biologique. } \\
\text { Utilisation de plaques } \\
\text { d'agar. } \\
\text { Acide tannique } \\
\text { couramment utilisé } \\
\text { comme témoin. }\end{array}$ \\
\hline
\end{tabular}

compris entre 3,5 et 7 (Jones et Mangan 1977). Ainsi, la figure 2 indique qu'il existe une relation étroite entre l'activité biologique et la teneur en TC (mesurée par la méthode HCL-butanol) au premier comme au deuxième cycle de végétation pour 3 variétés de sainfoin (Ambra, Esparcette, Villahoz) cultivées la même année sur le même site et pour une autre variété (Perly) cultivée en un site différent (Theodoridou 2010).

Certains auteurs ont montré que l'activité biologique est corrélée au $\mathrm{mDP}$ (Porter et Woodruffe 1984, Sarni-
Marchado et al 1999, Cheynier 2005, Frazier et al 2010). Mangan (1988) ainsi qu'Aerts et al (1999) ont trouvé que l'activité biologique augmentait avec le mDP jusqu'à une certaine valeur, puis lorsque le mDP continue d'augmenter, l'encombrement de la molécule de TC serait trop important pour que les TC réagissent avec les protéines. Ces résultats obtenus sur des feuilles de thé et du raisin, sont à l'encontre de ceux obtenus sur 3 variétés de sainfoin récoltées à des stades jeunes au premier cycle (Theodoridou et al 2011a) pour lesquelles l'activité biologique a tendance à diminuer lorsque le $\mathrm{mDP}$ de polymérisation augmente (figure 3 ).

D'après Aerts et al (1999), une teneur élevée en PD augmenterait la possibilité de former des complexes avec les protéines ce qui contribuerait à une augmentation de l'activité biologique. Or, comme pour le $\mathrm{mDP}$, cette relation n'a pas été retrouvée pour les 3 variétés de sainfoin étudiées par Theodoridou et al (2011a), l'augmentation observée du rapport $\mathrm{PD} / \mathrm{PC}$ n'étant pas liée à une augmentation de l'activité biologique. 
Figure 2. Relation entre l'activité biologique et la teneur en TC mesurée par la méthode HCL-butanol pour différentes variétés de sainfoin (d'après Theodoridou et al 2010, 2011a).

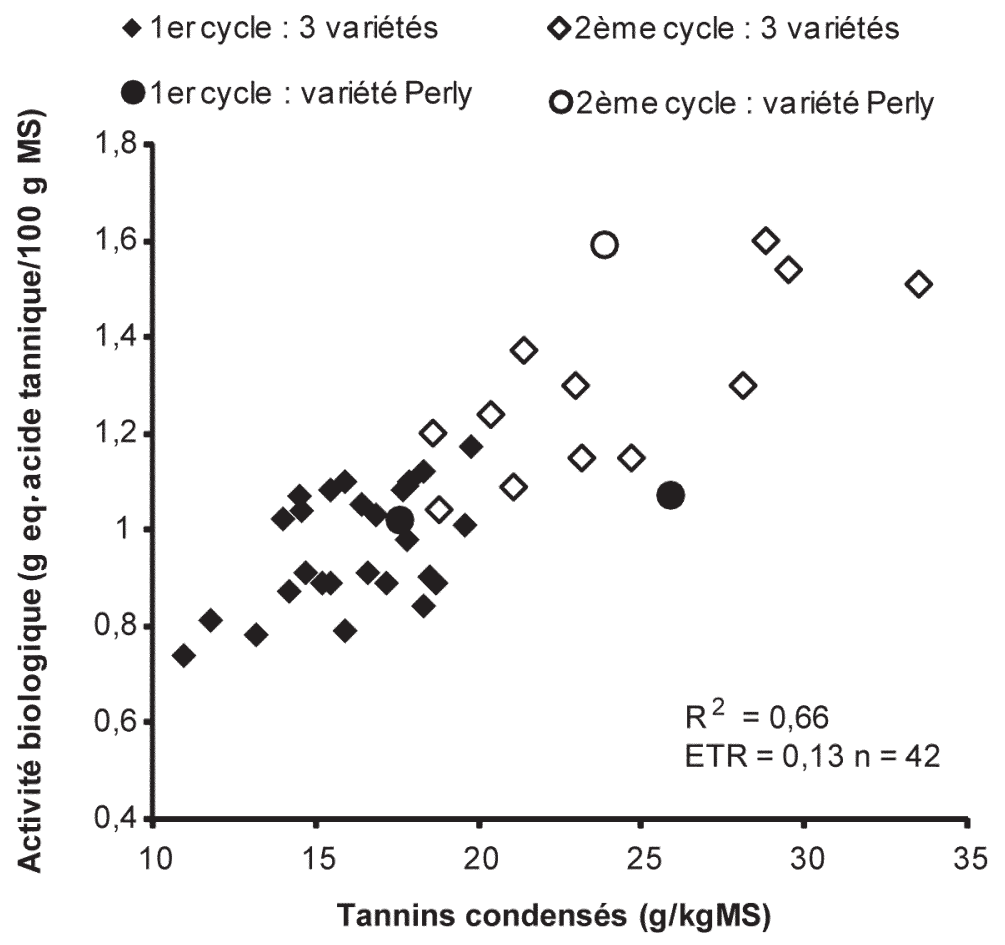

Figure 3. Relation entre l'activité biologique des TC et le degré de polymérisation (mDP) pour différentes variétés de sainfoin (d'après Theodoridou et al 2010, 2011a).

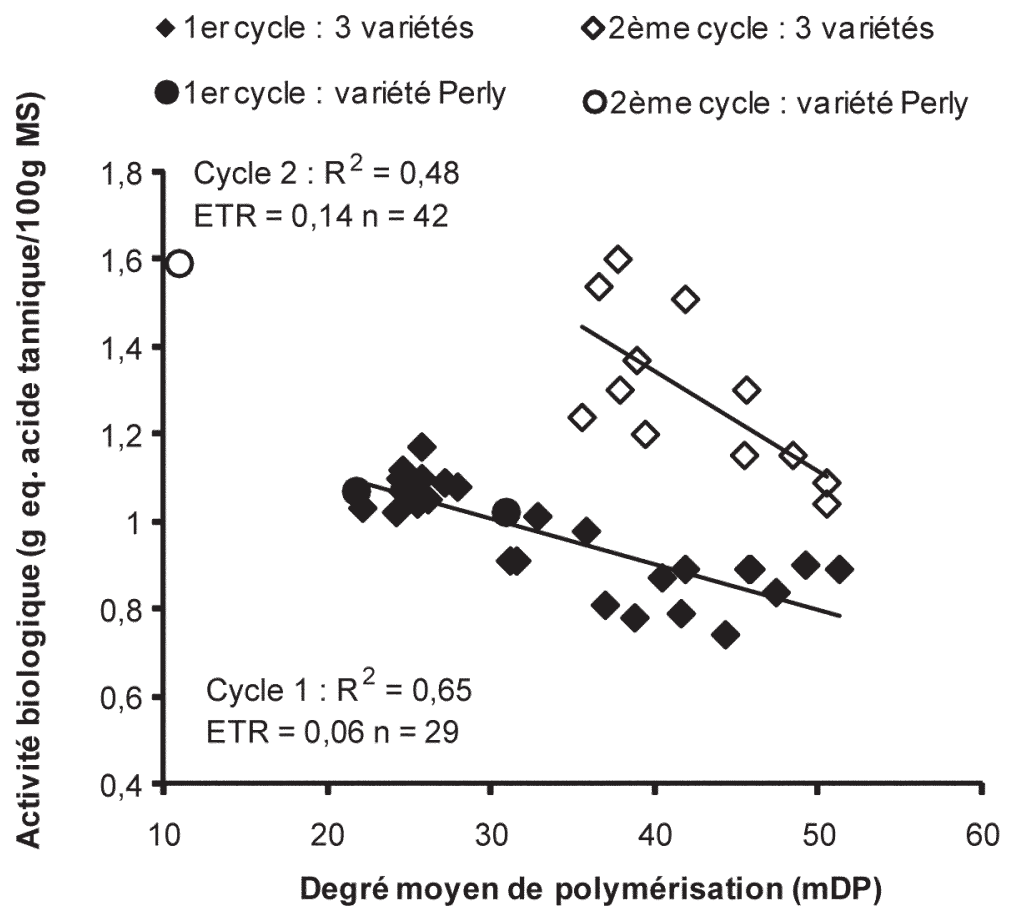

\section{4 / Facteurs de variation de la teneur et de l'activité des tannins condensés}

\section{a) Espèces et variétés}

La teneur en $\mathrm{TC}$ et le rapport $\mathrm{PD} / \mathrm{PC}$ peuvent présenter des différences plus ou moins marquées selon les variétés pour une même espèce. Ainsi pour 7 variétés de lotier corniculé récoltées à la mi-floraison, Hedqvist et al (2000) rapportent des valeurs de $\mathrm{PD} / \mathrm{PC}$ variant entre $16 / 84$ et $33 / 67$ et des teneurs en TC, mesurées par la méthode
HCL-butanol, allant de 0,2 à 1,7\% de la MS. Dans une autre étude, Marais et al (2000) observent des variations du rapport $\mathrm{PD} / \mathrm{PC}$ de $36 / 64$ à 93/7 pour différentes variétés de sainfoin mais n'indiquent pas les teneurs en TC. Les travaux de Theodoridou et al (2011a), montrent des différences significatives pour la teneur en TC mesurée par thiolyse $(0,42$ à $0,60 \%$ de la $\mathrm{MS})$, le $\mathrm{mDP}(32,5$ à 36,2$)$ et le rapport $\mathrm{PD} / \mathrm{PC}$ (67/33 à 68/32) entre 3 variétés de sainfoin cultivées sur le même lieu au premier cycle de végétation. En revanche, ces différences entre variétés ne sont pas significatives au deuxième cycle de végétation.

b) Stade de développement de la plante

Avec l'évolution du stade de développement la proportion de feuilles dans la biomasse de la plante entière diminue. Cela laisse supposer, les feuilles étant plus riches en TC que les tiges, que la teneur en TC diminue comme l'ont observé Borreani et al (2003), avec du sainfoin et du sulla, et Pilluza et al (2000) avec du sulla. Les résultats obtenus avec le sainfoin, variété Perly, récolté à des stades et des cycles différents par Theodoridou et al (2010) indiquent peu de variations de la teneur en TC. A l'inverse, Bell et al (1992), avec des feuilles de coton, Koupai-Abyazani et al (1993) avec du sainfoin, Theodoridou et al (2011a), avec 3 variétés de sainfoin, observent une augmentation de la teneur en TC avec le stade de développement. Ces résultats, apparemment contradictoires avec ceux rapportés plus haut, peuvent néanmoins s'expliquer: pendant la période de croissance, les plantes produisent une quantité importante de biomasse, et la synthèse des composés phénoliques est faible alors que pendant la floraison, la croissance est ralentie et le carbone serait disponible pour la synthèse des TC (Iason et al 1993).

L'élévation du rapport $\mathrm{PD} / \mathrm{PC}$ et du $\mathrm{mDP}$ avec le stade de développement en relation avec une diminution de l'activité biologique (figure 3) est contradictoire avec les résultats de la bibliographie obtenus sur d'autres types de végétaux (raisin, feuilles de thé, cf. § 1.3). Cela pourrait s'expliquer par le fait que la mesure de l'activité biologique ne porte que sur les tannins extractibles dont la quantité diminue avec la maturité de la plante (Gea et al 2011).

Les teneurs en TC et l'activité biologique plus importantes au deuxième cycle de végétation par rapport au premier peuvent s'expliquer par un rapport feuilles/tiges plus élevé au deuxième cycle (Theodoridou et al 2011a) et par des conditions environnementales 
(photopériode et température moyenne plus importantes) différentes de celles du premier cycle.

\section{c) Organes de la plante}

Les TC peuvent s'accumuler dans différents tissus (bourgeons, racines, graines, tiges). Selon Bell et al 1992, la majorité des tannins se trouvent dans les jeunes feuilles des plantes mais des teneurs élevées peuvent se trouver dans les fleurs et les fruits (Lees et al 1995, Frutos et al 2002, Gebrehiwot et al 2002). Les TC des fleurs de trèfle violet sont constitués principalement par de la PC alors que ceux des fleurs du trèfle blanc seraient constitués principalement par de la PD (Sivakumaran et al, 2004). Pour le sainfoin (variété Perly), les effets des TC sur la dégradation de l'azote dans le rumen sont plus importants pour les feuilles que pour les tiges en liaison avec leur concentration, leur activité biologique et leur rapport PD/PC plus élevés (Theodoridou et al 2010).

\section{d) Mode de conservation du fourrage}

L'intérêt d'utiliser des légumineuses à tannins conservées sous forme d'ensilage réside dans le fait que les TC capables de se fixer aux protéines conduisent à une moindre hydrolyse de ces dernières pendant le stockage et à une bonne conservation de l'ensilage sans ajout de conservateur, comme l'ont montré Albrecht et Muck (1991) et Theodoridou et al (2012).

Par ailleurs, l'activité biologique des TC est plus faible dans les ensilages que dans le fourrage vert correspondant, pour du sainfoin étudié à deux stades de développement (fin floraison au premier cycle de végétation et début floraison au deuxième cycle de végétation). Cela s'explique par des concentrations en TC extractibles et non extractibles plus faibles dans l'ensilage (Theodoridou et al 2011b). Toutefois, ces résultats sont en désaccord avec ceux obtenus par Minneé et al (2002) avec du lotier corniculé et du sulla, et par Scharenberg et al (2007) avec du sainfoin. Ces auteurs ne rapportent pas de modifications de teneur en TC dans l'ensilage par rapport au fourrage vert. Cependant, ils constatent que la teneur en tannins libres est réduite dans l'ensilage. Cette altération se ferait au cours de la mise en silo, qui cause une rupture cellulaire et la libération des TC, ceux-ci pouvant se combiner à d'autres molécules (Minneé et al 2002).

Pour le foin, la perte des feuilles et l'exposition au soleil peuvent expliquer la diminution de la teneur en TC au cours de la fenaison du sainfoin (Borreani et al 2003, Aufrère et al 2008).

\section{e) Environnement pédoclimatique}

Le stress hydrique induit des augmentations de teneur en TC (Anuraga et al 1993), mais la répartition entre les tannins libres et liés ou non aux protéines dépend du stress subit durant la croissance de la plante (Iason et al 1995). Il existe aussi une relation positive entre l'intensité lumineuse et la quantité de polyphénols produits dans une plante (Mole et Waterman 1987).

Lees et al (1994) observent une augmentation de la teneur en TC dans le lotier pédonculé Cav. (lotus uliginosus) quand la température est de $30^{\circ} \mathrm{C}$ comparée à $20^{\circ} \mathrm{C}$, alors qu'il n'en est pas de même pour le lotier corniculé.

Des teneurs plus élevées en TC ont été également observées sur des sols acides peu fertilisés alors que celles-ci sont plus faibles sur des sols très fertilisés (Kelman et Tanner 1990).

En conclusion de cette première partie, les relations entre la teneur, la structure et la capacité des TC à se lier aux protéines sont complexes et les résultats dépendent en partie de l'espèce végétale, du stade de développement de la plante, de son mode de conservation, de son environnement, mais aussi des méthodes de dosages utilisées qui ne mesurent pas toutes les mêmes entités. La structure des TC est un vaste domaine de recherche et a été peu étudiée jusqu'à maintenant sur les fourrages. Lorsqu'elle a été mesurée, la relation entre la structure chimique et l'activité biologique n'est pas simple à interpréter (Waghorn 2008, Theodoridou 2010, Theodoridou et al 2010, 2011a, b et 2012). En conséquence, dans ce texte, la structure des TC ne pourra pas toujours être prise en compte pour expliquer l'effet des TC sur les paramètres de la valeur alimentaire.

\section{2 / Valeur alimentaire des légumineuses contenant des tannins condensés}

\section{1 / La composition chimique}

Le stade de développement et le rapport feuilles/tiges dans la plante entière sont les principaux facteurs qui font varier la composition chimique (teneur en azote, constituants pariétaux, sucres solubles...) des plantes fourragères. Des résultats comparant intra-expérimentation la composition chimique de légumineuses avec et sans TC sont rapportés dans le tableau 1.

Contrairement aux légumineuses classiques (trèfle, luzerne...), les légu- mineuses contenant des TC ont fait l'objet de peu de programmes de sélection, ce qui peut expliquer une teneur en Matières Azotées Totales (MAT) toujours inférieure à celle du fourrage correspondant sans tannin. Dans une étude comparant 3 variétés de sainfoin et une variété de luzerne cultivées dans un même lieu, à $870 \mathrm{~m}$ d'altitude, la même année, Theodoridou et al (2011a) ont mesuré en utilisant l'échelle de Kalu et Fick (1981) un développement phénologique, plus précoce du sainfoin au premier cycle de végétation que celui de la luzerne. Borreani et al (2003) expliquent la diminution plus rapide de la teneur en MAT au premier cycle de végétation pour le sainfoin que pour la luzerne par une baisse plus rapide du rapport feuille/tige à partir du début de la floraison.

La méthode de Van Soest et Robertson (1980) permettant de déterminer la teneur en constituants pariétaux (NDF) n'est pas applicable telle quelle sur les fourrages riches en tannins. En effet, ces derniers peuvent se lier aux protéines ou se complexer avec les constituants pariétaux, ce qui entraîne un biais dans les résultats (Makkar et al 1995, Pagan et al 2009). Aussi, un traitement avec du sulfite de sodium doit être appliqué sur les échantillons pour éliminer les tannins. Nous avons donc appliqué le cas échéant aux valeurs de la bibliographie une relation établie dans notre laboratoire pour corriger les teneurs en NDF. La teneur en constituants pariétaux est proche entre les fourrages, avec et sans TC. A même stade de végétation, la teneur en NDF du sainfoin est plus faible, ou similaire à celle de la luzerne (Aufrère et al 2008, Theodoridou et al 2011a). Une particularité des fourrages contenant des TC est leur richesse en glucides solubles (tableau 1) dont la teneur est beaucoup plus élevée que celle des légumineuses classiques (luzerne, trèfle) (Waghorn et al 2002, Minneé et al 2002, Theodoridou et al non publié), ce qui favorise leur aptitude à la conservation en ensilage.

\section{2 / Mise en évidence des effets digestifs et nutritionnels des tan- nins condensés contenus dans les fourrages}

L'effet des TC contenus dans un fourrage peut être estimé par comparaison avec un fourrage ne contenant pas de TC. Toutefois, il n'est généralement pas possible de conduire cette comparaison à composition chimique comparable pour les autres constituants des fourrages (cf. § 2.1). Les méthodes qui inhibent les TC sont donc préférables. L'utilisation du PolyEthylène Glycol (PEG) qui en se combinant aux TC 
Tableau 1. Composition chimique (Teneur en Matières Azotées Totales (MAT), Glucides Solubles (GS), constituants pariétaux (NDF), en g/kg MS) pour quelques fourrages contenant ou non des tannins condensés (TC). FV : Fourrage Vert, Ens : Ensilage.

\begin{tabular}{|c|c|c|c|c|c|}
\hline \multirow[t]{2}{*}{ Fourrages } & \multicolumn{4}{|c|}{ Composition chimique ( $\mathrm{g} / \mathrm{kg} \mathrm{MS}$ ) } & \multirow[t]{2}{*}{ Références } \\
\hline & MAT & GS & NDF & $\mathrm{TC}$ & \\
\hline Sulla (FV) & 175 & 232 & 202 & 68 & Waghorn et al (2002) \\
\hline Luzerne séchée & 240 & 152 & 314 & 0 & \\
\hline Ray-grass/trèfle blanc (FV) & 185 & 135 & 444 & 0 & \\
\hline Trèfle violet (FV) & 244 & 111 & 342 & 3 & Waghorn et al (2002) \\
\hline Sulla (FV) & 197 & 176 & 226 & 35 & \\
\hline Lotier pédonculé (FV) & 264 & 113 & 293 & 53 & \\
\hline Luzerne séchée & 220 & 150 & 352 & 0 & \\
\hline Sainfoin (FV, mi-végétatif) & 223 & 148 & 208 & 16,1 & Theodoridou et a/ non publié \\
\hline Sainfoin (FV, début bourgeonnement) & 98 & 122 & 443 & 14,2 & \\
\hline Luzerne (FV, végétatif précoce) & 220 & 92 & 198 & 0 & \\
\hline Luzerne (FV, végétatif tardif) & 158 & 52 & 445 & 0 & \\
\hline Lotier corniculé (FV) & 196 & 84 & 346 & 34 & Wang et al (1996) \\
\hline Luzerne (FV) & 261 & 64 & 299 & 0 & \\
\hline Lotier (Ens., fin bourgeonnement) & 218 & 50 & 329 & non mesuré & Fraser et al (2000) \\
\hline Sainfoin (Ens., fin floraison & 121 & 29 & 404 & non mesuré & \\
\hline Trèfle violet (Ens., floraison) & 213 & 90 & 345 & non mesuré & \\
\hline Luzerne (Ens., début floraison) & 182 & 5,5 & 480 & 0 & \\
\hline
\end{tabular}

supprime leurs effets, sans affecter les enzymes microbiennes et digestives (Jones et Mangan, 1977, Barry et Manley 1986) permet d'étudier l'effet des TC sur les paramètres digestifs et nutritionnels par rapport au même fourrage étudié sans ajout de PEG. Toutefois l'utilisation du PEG nécessite des précautions. La quantité de PEG, administré aux animaux sous forme liquide, doit être suffisante pour que tous les TC soient inactivés, mais une dose excessive de PEG peut augmenter le taux de passage de la phase liquide à la sortie du rumen et provoquer des diarrhées (Schiller et al 1988). Des doses plus ou moins importantes ont été utilisées pour l'étude des effets des TC du sainfoin (100 g/jour de PEG 4000 pour Scharenberg et al 2007, $160 \mathrm{~g} /$ jour de PEG 3500 pour Bermingham et al 2001), du sulla (120 g/jour de PEG 3500 , Bermingham et al 2001) ou encore du lotier pédonculé (75 à $100 \mathrm{~g} /$ jour de PEG 3350, Barry et Manley 1985). Les essais réalisés par Theodoridou (2010), Theodoridou et al (2010, 2012), l'ont été avec $60 \mathrm{~g} /$ jour de PEG 4000, selon les recommandations de Barry et Forss (1983) soit 1,7 g PEG/g TC, ce qui n'a pas provoqué de diarrhées chez les animaux, sans que l'on puisse toutefois affirmer de façon certaine que la totalité des TC était inactivée.

\section{3 / L'ingestibilité}

En utilisant le PEG pour inactiver les TC, il apparaît que ces derniers ne modifieraient pas significativement l'ingestion de fourrages verts de sainfoin $(\mathrm{TC}=38$ à $77 \mathrm{~g} / \mathrm{kg}$ de $\mathrm{MS}$, Bermingham et al 2001, Scharenberg et al 2007), de sulla $(\mathrm{TC}=64$ à $72 \mathrm{~g} / \mathrm{kg}$ de MS, Terrill et al 1992b, Stienezen et al 1996, Douglas et al 1999, Bermingham et al 2001), de lotier corniculé $(\mathrm{TC}=20$ à $35 \mathrm{~g} / \mathrm{kg}$ de MS, Waghorn et al 1987, Wang et al 1994, 1996) ou pédonculé $(\mathrm{TC}=53 \mathrm{~g} / \mathrm{kg}$ de $\mathrm{MS}$, Waghorn et al 2002). En revanche, Barry et Duncan (1984) comme Waghorn et al (1994a), observent un effet négatif des TC du lotier pédonculé sur son ingestibilité à des teneurs en TC respectivement de $63 \mathrm{~g} / \mathrm{kg}$ de MS et de $55 \mathrm{~g} / \mathrm{kg}$ de MS. Toutefois, Barry et Mc Nabb (1999) estiment que la faible ingestibilité du lotier pédonculé ne serait pas due seulement à une teneur en TC élevée dans cette plante. En effet, la diminution d'ingestibilité observée avec du lotier pédonculé ayant une teneur en TC de $55 \mathrm{~g} / \mathrm{kg}$ MS n'a pas été retrouvée avec le lotier corniculé à teneur en TC voisine (34 à $44 \mathrm{~g} / \mathrm{kg} \mathrm{MS})$. La structure des tannins, différente entre ces deux espèces, riches en PC pour le lotier corniculé et riches en PD pour le lotier pédonculé (Foo et al 1997), pourrait expliquer les effets différents sur l'ingestibilité
(Waghorn 2008). Bien que les TC du sainfoin (Mueller-Harvey 2006, Theodoridou et al 2010) et du sulla (Tibe et al 2011) présentent une structure analogue à ceux du lotier pédonculé, les résultats de la bibliographie n'indiquent pas une ingestibilité plus faible pour ces deux plantes. En revanche, la comparaison entre plusieurs types de Calliandra calothyrus (mimosa) montre que lorsque les unités PD sont dominantes dans la fraction de tannin extractible, l'ingestion est augmentée (Lascano et al 2001). Ces résultats indiquent que le lien entre la teneur en TC, leur structure et leur effet sur l'ingestibilité n'est pas complètement élucidé. D'après Frutos et al (2004), trois mécanismes pourraient expliquer l'effet négatif d'une teneur élevée en TC sur l'ingestion volontaire : i) une réduction de l'appétence, $i$ i) une diminution de la vitesse de digestion et iii) des modifications des fermentations microbiennes dans le rumen.

Lorsque les légumineuses à tannins sont comparées à des légumineuses n'en contenant pas, Parker et Moss (1981) et Aufrère et al (2008) n'ont pas mis en évidence de différences d'ingestibilité entre des fourrages verts de sainfoin et de luzerne. Ulyatt et Egan (1979) ont même observé une ingestibilité plus élevée avec le sainfoin qu'avec le trèfle blanc. Terrill et al (1992a), notent une 
Figure 4. Relation entre la digestibilité de la Matière Organique (dMO) et la Digestibilité pepsine-cellulase (Dcellms).

- Sainfoin mesuré ad libitum (•) (Aufrère et al 2008, Aufrère et al non publié) ou en quantité limitée $(\diamond)$, (Theodoridou et al 2010)

- Luzerne et trèfle violet $(\boldsymbol{\Delta})$.

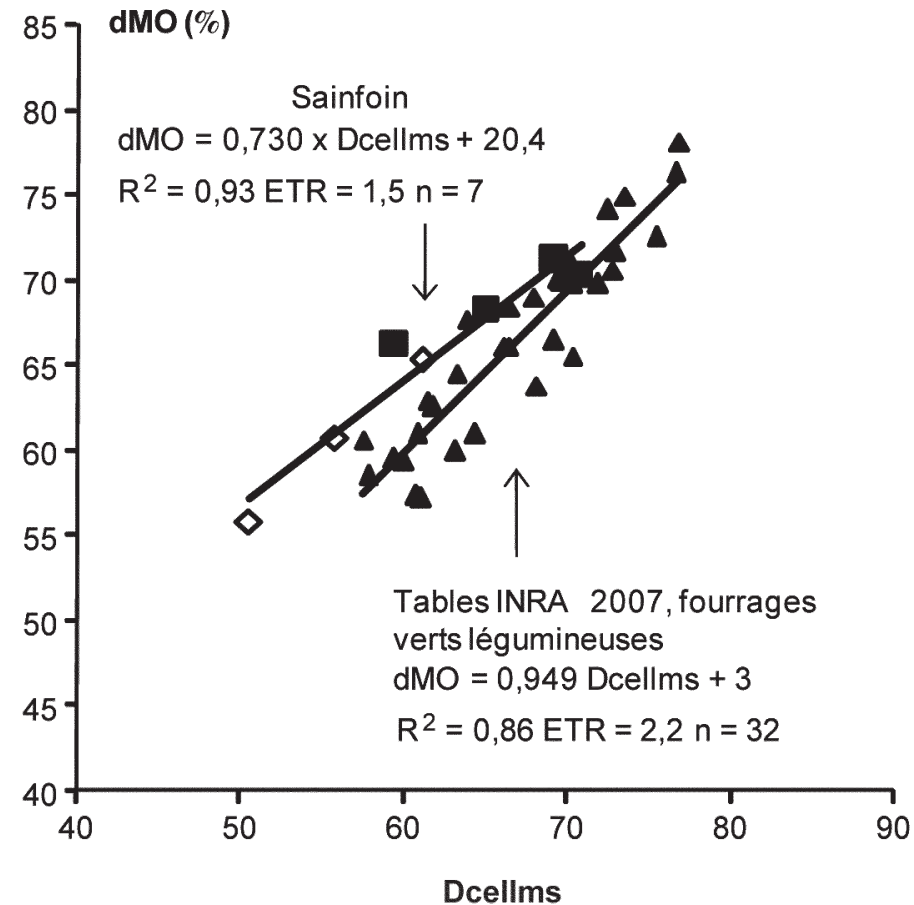

ingestibilité plus importante avec du sulla qu'avec un mélange de graminées pérennes et Waghorn et al (2002) avec du sulla comparé à un mélange raygrass trèfle blanc. Contrairement aux observations de Douglas et al (1995), Wang et al (1996) n'ont pas mesuré de différence d'ingestibilité entre le lotier corniculé et la luzerne tandis que Fraser et al (2000) ont mesuré une ingestibilité plus élevée avec l'ensilage de lotier corniculé qu'avec celui de luzerne.

\section{4 / La digestion de la matière organique}

a) Effet des tannins condensés sur la digestibilité dans l'ensemble du tube digestif

Waghorn et al (1987) ainsi que Wang et al (1996) n'ont pas trouvé d'effet des TC sur la digestibilité de la Matière Organique $(\mathrm{dMO})$ du lotier corniculé contenant 2 à $3 \%$ de tannins actifs, de même que Waghorn et al (1994a) sur la digestibilité des constituants pariétaux du lotier pédonculé plus riche en TC. En revanche, Barry et Duncan (1984) ainsi que Barry et al (1986) observent une augmentation de la dMO pour le lotier pédonculé lorsque les TC sont inactivés par le PEG (PEG 3350, 75 à $100 \mathrm{~g} /$ jour). Stienezen et al (1996) obtiennent une augmentation de 2 points de digestibilité avec du sulla en présence de PEG (PEG 3000, 200 g/jour) alors que Terrill et al (1992b) n'en n'observent pas (PEG 3350, 40 g/jour).
En utilisant du PEG pour inactiver les TC ou en comparant avec de la luzerne, Kraiem et al (1990), Aufrère et al (2008) et Theodoridou et al (2010, 2012) (PEG 4000, 60 g/jour) n'ont pas montré d'effet négatif des TC sur la dMO du sainfoin récolté en vert ou conservé sous forme d'ensilage mifané. Au contraire, Scharenberg et al (2007) mettent en évidence un effet négatif des TC sur la dMO du sainfoin séché ou ensilé (PEG 3500, 160 g/jour), et Bermingham et al (2001) un effet négatif sur la dMO du fourrage vert de sainfoin et de sulla (PEG 3500, 120 et $160 \mathrm{~g} /$ jour respectivement pour le sainfoin et le sulla).

Les mêmes discordances sont observées dans des études in vitro. Theodoridou et al (2011b), n'ont pas constaté d'augmentation de dMO en présence de PEG pour le fourrage vert de sainfoin. A l'inverse Karabulut et al (2006) ont remarqué une augmentation de la digestibilité du lotier corniculé en présence de PEG.

Les effets variables des TC sur la dMO s'expliquent en partie par la teneur en TC, comme dans l'étude de Barry et Manley (1984) et dans celle de Chiquette et al (1988) qui comparent respectivement du lotier pédonculé et corniculé à deux teneurs en TC. De plus lorsque le PEG est utilisé en plus faible quantité, aucun effet n'est observé sur la dMO ce qui pourrait suggérer que tous les TC n'ont pas été inactivés, mais ces résultats correspondent aussi à de faibles teneurs en TC de 1,8 à 2,6\% de MS, déterminées par une méthode $\mathrm{HCl}$-butanol simplifiée (Theodoridou 2010).

b) Effet sur la dégradation de la Matière Sèche (MS) dans le rumen et sur la production de méthane

Alors que l'on n'observe pas d'effet des TC sur la dMO de la plante entière de sainfoin, les TC diminuent la dégradation in situ dans le rumen de la MS des feuilles et des tiges de sainfoin récoltées en vert (Theodoridou et al 2010a) et de l'ensilage mi-fané, (Theodoridou et al 2012). Ce résultat a également été observé par Aufrère et al (2008) qui trouvent une dégradation plus faible du fourrage vert de sainfoin dans le rumen que celle de la luzerne aux mêmes stades de végétation alors qu'aucune différence n'a été observée sur la dMO. Cela pourrait s'expliquer par le fait que la diminution de la dégradation de la MS dans le rumen en présence de TC est compensée par une plus forte digestion dans l'intestin une fois les complexes TC-protéines solubilisés, conduisant finalement à une absence d'effet sur la digestibilité totale de la matière organique.

Les TC auraient une activité antiméthanogène soit en inhibant les bactéries méthanogènes ou indirectement par l'inhibition des protozoaires (Animut et al 2008 chez la chèvre, Bhatta et al 2009, Jayanegara et al 2009, Patra et Saxena 2011). Des études in vitro sur le sainfoin seul (Mc Mahon et al 2000, Theodoridou et al 2011b) ou en association avec du ray-grass ou du dactyle (Niderkorn et al 2011) montrent une diminution de la production de méthane en présence de ce fourrage. Des effets semblables ont été observés in vivo avec le sainfoin (Tamminga et al $2007 \mathrm{chez}$ le bovin, Waghorn 2008), le lotier corniculé (Woodward et al 2004 chez la vache laitière) et pédonculé (Waghorn et al 2002, Tavendale et al 2005), le sulla (Woodward et al 2002 chez la vache laitière). En revanche, les résultats in vitro de Guglielmelli et al (2011) avec du foin de sainfoin indiquent que les TC du foin ne provoquent pas de diminution de méthane.

c) Relation entre la digestibilité des légumineuses à tannins et la digestibilité enzymatique "pepsine-cellulase»

La dMO des fourrages, premier facteur de variation de leur valeur énergétique, peut être prévue par une méthode enzymatique (digestibilité pepsine-cellulase, Aufrère et al 2007). L'équation de prévision de la dMO des fourrages verts de légumineuses proposée en 2007 
a été établie sur 32 échantillons de luzerne et de trèfle violet et n'intègre pas de légumineuses riches en TC.

La méthode pepsine-cellulase utilisée pour prévoir au laboratoire la dMO des fourrages a été testée sur des échantillons de sainfoin dont la dMO chez le mouton avait été mesurée in vivo à l'INRA de Theix entre 2004 et 2010 (figure 4). La précision de l'équation de prévision obtenue est $\left(\mathrm{R}^{2}=0,93, \mathrm{ETR}=\right.$ $1,5, \mathrm{n}=7$ ). Ramirez-Restrepo et al (2006) ont montré également qu'il était possible d'utiliser des enzymes cellulolytiques et hemi-cellulolytiques pour prévoir la $\mathrm{dMO}$ de plantes fourragères à tannins comme le lotier corniculé.

d) Relation entre la digestibilité des légumineuses à tannins et leur teneur en constituants pariétaux

Pour les trois variétés de sainfoin cultivées la même année dans un même lieu (Theodoridou et al 2011a), la dMO estimée à partir de la digestibilité pepsine-cellulase est liée de façon très étroite à la teneur en NDF $\left(\mathrm{R}^{2}=0,96, \mathrm{ETR}=\right.$ $1,2, \mathrm{n}=39$ ). On observe que la liaison entre la dMO prévue à partir de la digestibilité pepsine cellulase et la teneur en NDF n'est pas différente pour le sainfoin et la luzerne. Ce résultat est en accord avec ceux de Aufrère et al (2008) comparant sainfoin et luzerne et avec ceux de Kraiem et al (1990) qui n'observent pas de différences de dMO entre luzerne, lotier corniculé et sainfoin récoltés au même stade de végétation.

Toutefois, lorsqu'on rassemble les différentes mesures de $\mathrm{dMO}$ mesurée in vivo du sainfoin disponibles dans la littérature et qu'on les met en relation avec la teneur en NDF, on observe quelques différences entre variétés, ou entre études. Les résultats de Scharenberg et al (2007), Aufrère et al (2008), Arrigo (2009), s'inscrivent bien dans la relation obtenue pour les trois variétés.

De même, on observe des différences entre espèces de légumineuses à tannins

Figure 5. Influence de la teneur en constituants pariétaux (NDF) de différentes légumineuses à tannins sur la digestibilité de la Matière Organique du fourrage (dMO).

Sainfoin : $\bullet$ ) 3 variétés (Ambra, Villahoz, Esparcette) (Theodoridou et al 2011a); (•) Variété Perly (Theodoridou et al 2010, 2011b) ; Fourrage vert (৯Tables INRA 2007, - Aufrère et al 2008).

Lotier pédonculé $(\triangle)$ : Barry et Duncan 1984, Barry et Manley 1986, Barry et al 1986, Waghorn et al 1994a, Waghorn et Shelton 1995.

Lotier corniculé (O) : Kraiem et al (1990), Tables méditerranéennes (1990), Douglas et al (1999).

Sulla ( $\square$ ) : Tables méditerranéennes (1990), Douglas et al (1999), Molle et al (2009).

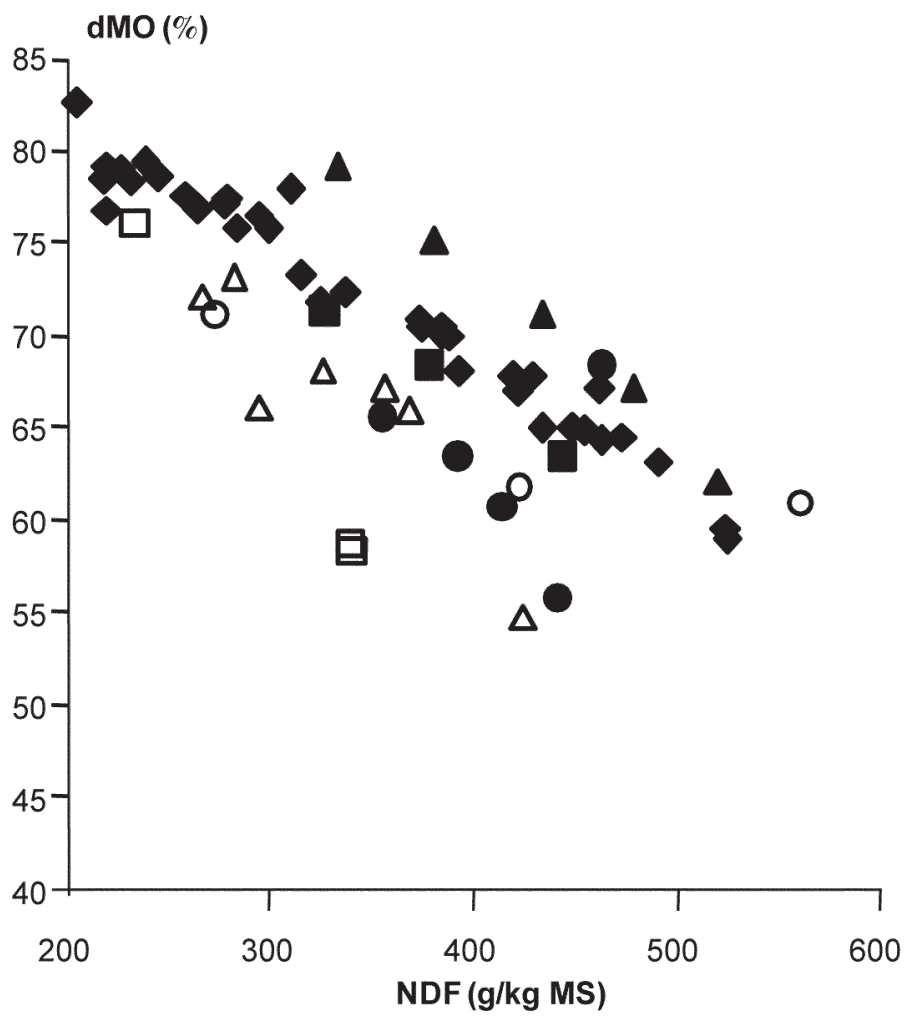

dans la liaison entre NDF et dMO (figure 5). Globalement, à même teneur en NDF, les autres fourrages contenant des TC (lotier corniculé ou pédonculé, sulla) semblent avoir une digestibilité plus faible que celle estimée pour les trois variétés de sainfoin. La part des modifications dues à l'environnement ou celles dues à la teneur en TC ne peut être quantifiée avec précision dans cette comparaison. La faible digestibilité d'un échantillon de lotier pédonculé (Barry et Duncan 1984) pourrait être liée à une teneur élevée en TC (106 g/kg MS).

\section{5 / La digestion des matières azotées}

a) Digestibilité des matières azotées dans l'ensemble du tube digestif.

La digestibilité des Matières Azotées Totales (dMAT) des fourrages verts ou conservés contenant des TC est faiblement reliée à leur teneur en MAT $\left(\mathrm{R}^{2}=\right.$ $0,25, \mathrm{n}=50$ ). En effet, pour ces fourrages, la teneur et l'activité plus ou moins importante des TC entraînent des variations importantes de dMAT. L'effet négatif des TC sur la dMAT est bien mis en évidence dans les expériences dans lesquelles du PEG a été ajouté à la ration (figure 6a).

La dMAT augmente en présence de PEG de 5 à 26 points selon l'espèce étudiée (figure 6a). Ainsi, à même teneur en MAT, la dMAT est plus faible pour le lotier pédonculé que pour le lotier corniculé en relation avec la teneur et la structure des TC. L'effet des TC est variable d'une expérience à une autre pour un même fourrage, mais pour des teneurs en MAT comprises entre 180 et $250 \mathrm{~g} / \mathrm{kg} \mathrm{MS}$, la dMAT atteint un plateau en présence de PEG.

A même teneur en MAT, la dMAT des fourrages contenant des TC (figure $6 \mathrm{~b}$ ) est plus faible que celle de la luzerne ou de mélanges graminées-légumineuses. Dans cette comparaison la liaison entre la dMAT et la teneur en MAT est significative $\left(\mathrm{R}^{2}=0,52, \mathrm{ETR}=3,9 \mathrm{n}=16\right)$ pour les fourrages sans TC, alors qu'elle ne l'est pas pour les fourrages contenant des TC.

b) Dégradation des matières azotées dans le rumen

La diminution de la dégradation de l'azote dans le rumen des fourrages contenant des tannins est bien connue (Jones et Mangan 1977). Elle s'explique par la liaison tannins-protéines qui empêche les protéines de se dégrader. Min et al (2001) observent que cette liaison est influencée par la structure et le poids moléculaire des TC et des protéines, sans modification de la synthèse 
Figure 6. Relation entre la dMAT et la teneur en MAT pour différents fourrages contenant des TC (a) avec et sans ajout de PEG, (b) par comparaison avec la luzerne ou des mélanges graminées légumineuses.

a) Effet du PEG : Données issues de Barry et Duncan (1984), Barry et Manley (1984), Barry et al (1986), Waghorn et al (1987), Waghorn et al (1994b), Wang et al (1994, 1996), Waghorn et Shelton (1995, 1997), Stienezen et al (1996), Bermingham et al (2001), Scharenberg et al (2007), Theodoridou et al (2010, 2011).

b) Comparaison avec des fourrages sans TC : données issues de Harrison et al (1973), Egan et Ulyatt (1980), Parker et Moss (1981), Kraiem et al (1990), Scharenberg et al (2007), Aufrère et al (2008), Arrigo et Scharenberg (2008), Arrigo et Dohme (2009), Aufrère et al (non publié).

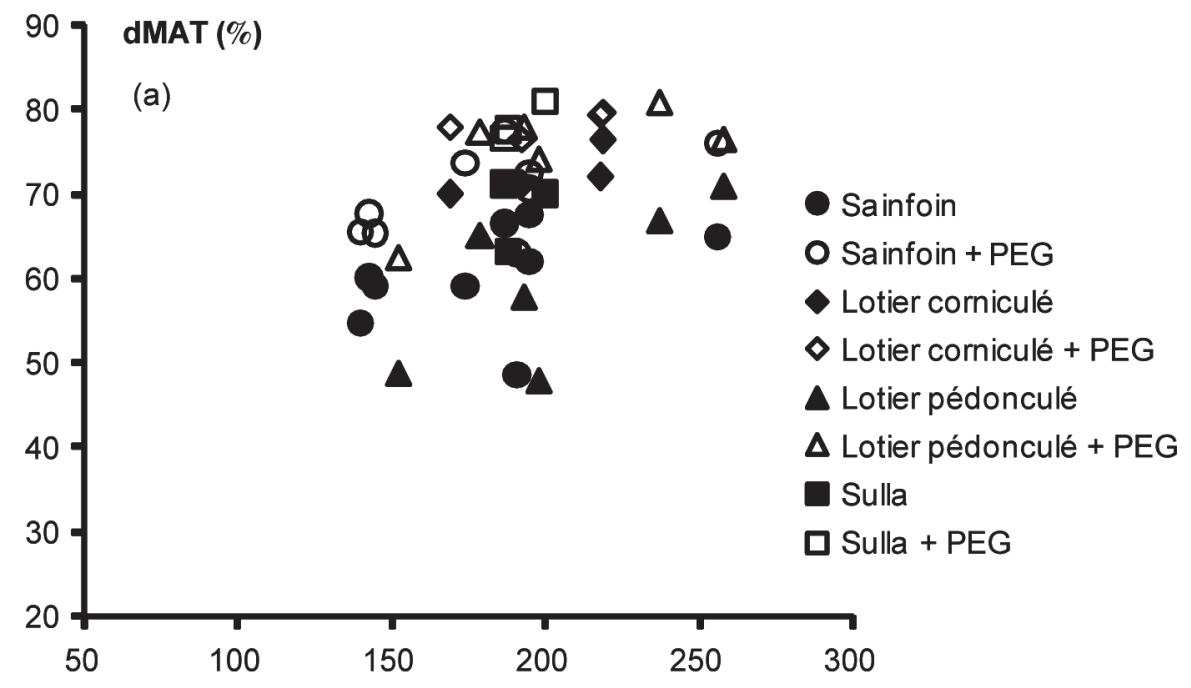

MAT (g/kg MS)

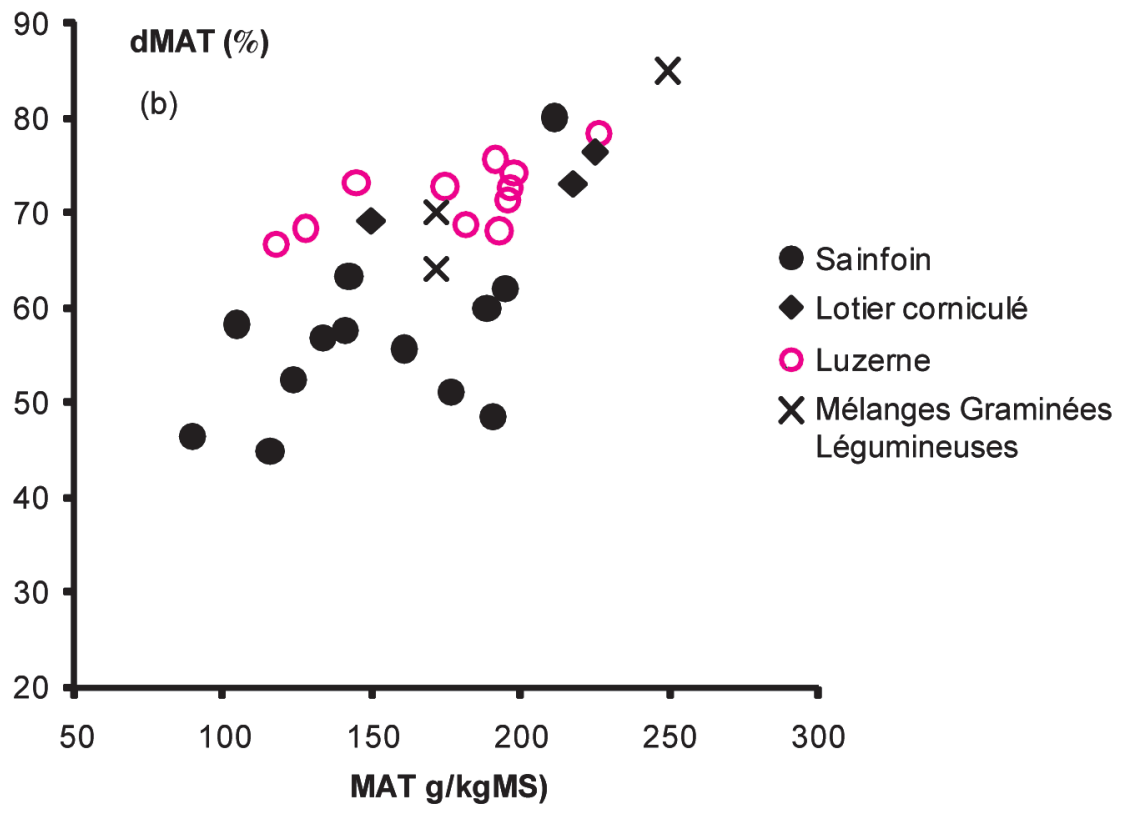

de protéines microbiennes dans le rumen. Les TC pourraient se lier aux protéines alimentaires, à la microflore ou aux enzymes microbiennes.

Hedqvist et al (2000) estiment que les tannins ont un effet sur la dégradation de l'azote et des parois même lorsque la teneur en tannins est faible. Les résultats d'Arrigo et Scharenberg (2008), dabilité des MAT en comparant un mélange fourrager (graminées, légumineuses, pissenlit) et du lotier corniculé récoltés au troisième cycle de végétation.

Avec du sainfoin, Theodoridou et al (2010, 2011b) observent in situ et in vitro des dégradabilités plus élevées de l'azote dans le rumen pour les tiges que pour les feuilles bien que la teneur en azote des tiges soit plus faible que celle des feuilles. Ces différences s'expliquent d'une part, par une teneur plus élevée en TC (mesurée par la méthode HCL-butanol et par thiolyse) et une activité biologique plus élevée des TC dans les feuilles, et d'autre part, par des localisations différentes de l'azote dans les tissus des tiges et des feuilles.

Les concentrations en azote total $(\mathrm{Nt})$ et en ammoniac $\left(\mathrm{NH}_{3}\right)$ retrouvées dans le jus de rumen reflètent la dégradation dans le rumen des fractions rapidement dégradables. Quels que soient le cycle de végétation et le stade de développement du sainfoin (Theodoridou et al 2010), les teneurs en $\mathrm{Nt}$ et $\mathrm{NH}_{3}$ présentent un pic, comme pour les fourrages classiques, $1,5 \mathrm{~h}$ après le repas du matin, et diminuent ensuite jusqu'à $6 \mathrm{~h}$ après le repas. In vivo et in vitro avec du fourrage vert de sainfoin, Theodoridou et al (2010 et 2011b) observent que l'évolution reste la même au cours de la journée, mais que les teneurs en $\mathrm{Nt}$ et $\mathrm{NH}_{3}$ sont plus élevées en présence de PEG qui inactive les TC. Toutefois, l'effet du PEG sur les teneurs en $\mathrm{Nt}$ et $\mathrm{NH}_{3}$ n'a pas été observé avec l'ensilage mifané de sainfoin (Theodoridou et al 2012) alors qu'il l'a été avec le sainfoin ensilé et déshydraté par Scharenberg et al (2007) et par Minneé et al (2002) avec du sulla et du lotier corniculé. Une diminution des tannins libres et une augmentation des TC liés aux parois durant la fermentation dans le silo pourrait entraîner une diminution de la quantité de tannins libres dans l'ensilage pouvant se combiner aux protéines (Minneé et al 2002).

c) Digestibilité des matières azotées dans l'intestin

Alors que la liaison tannins-protéines est favorisée dans le rumen et que les complexes tannins-protéines sont insolubles au $\mathrm{pH}$ du rumen, ces complexes sont hydrolysés dans la caillette et le début de l'intestin grêle à un $\mathrm{pH}$ inférieur à 3 (Jones et Mangan 1977).

A notre connaissance, il existe peu d'études de la digestion de l'azote des fourrages contenant des TC dans l'intestin. Aufrère et al (2008), par la technique des sachets mobiles, observent que la digestibilité des matières azotées, 
Figure 7. Excrétion d'azote dans les fèces et dans l'urine pour (a) le sainfoin et la luzerne récoltés au 1er cycle de végétation. (Aufrère et al 2008) ; (b) le fourrage vert et l'ensilage mi-fané de sainfoin au $2^{\text {ème }}$ cycle de végétation, en présence ou non de PEG. (Theodoridou et al 2010, 2011 b).

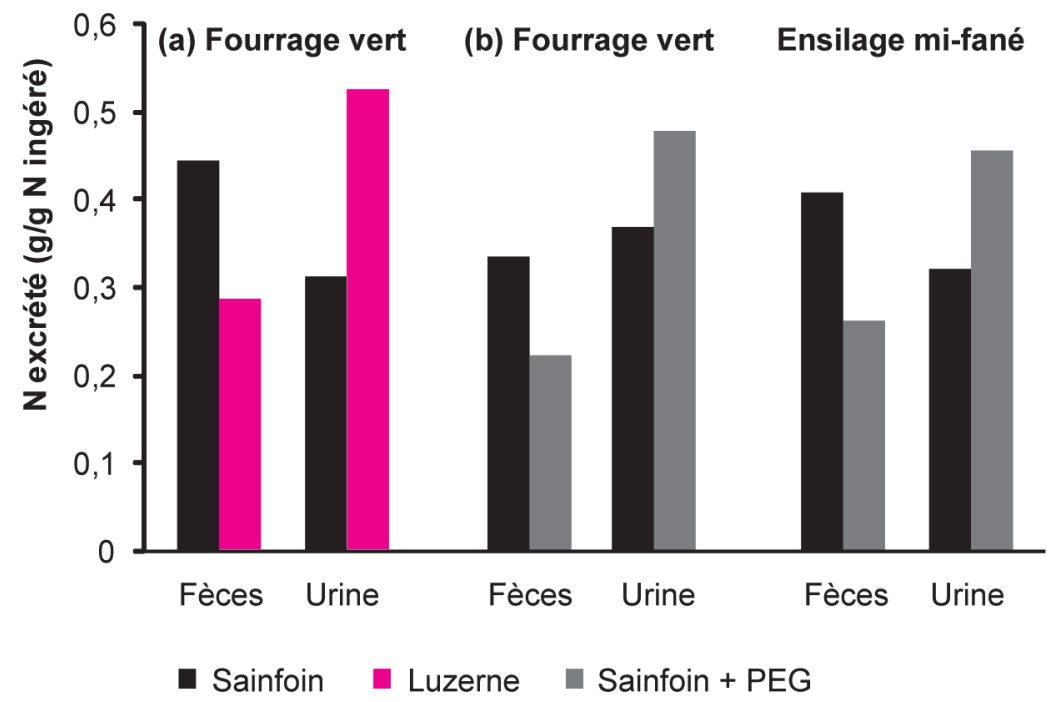

non dégradées dans le rumen, est faible dans l'intestin particulièrement pour le sainfoin par rapport à la luzerne. La comparaison du sainfoin avec la luzerne (Kraiem et al 1990) ou le trèfle blanc (Egan et Ulyatt 1980) indique une diminution semblable de la digestibilité intestinale de l'azote. En revanche, Kraiem et al (1990) ne trouvent pas de différence de digestion de l'azote entre le lotier corniculé et la luzerne, étudiés à même teneur en azote. Bermingham et al (2001) ont observé que la disparition de l'azote dans l'intestin était plus élevée pour le sulla que pour le sainfoin. Cela serait le résultat d'une plus faible teneur en azote et d'une plus faible activité des TC. Theodoridou et al (2010) observent que les MAT des feuilles de sainfoin sont plus digérées dans l'intestin que celles des tiges, en conséquence de leur moindre dégradabilité dans le rumen.

Bien que l'impact des TC sur la digestibilité intestinale soit encore mal connu (Patra et Saxena 2011), il semble que les TC inhibent la capacité des enzymes endogènes à hydrolyser les protéines en peptides et acides aminés (Waghorn 2008). D'après Min et al (2003), les TC peuvent se lier aux cellules bactériennes et à la rubisco mais les interactions TCbactéries seraient plus robustes que les interactions TC-protéines (Molan et al 2001). Par conséquent, les TC pourraient se lier aux enzymes extracellulaires inhibant leur activité de façon plus ou moins importante selon le type de TC et de fourrage : les TC du lotier corniculé et du sulla augmentent l'absorption des acides aminés au niveau de l'intestin alors que ceux du lotier pédonculé et du sainfoin ne produisent pas de modification de l'absorption. Les cau- ses de ces particularités pourraient en partie être dues à la différence de structure chimique des TC (Min et al 2003). Des travaux récents (Tibe et al 2011) indiquent que les TC extractibles du sulla seraient composés principalement de PD comme ceux du sainfoin et du lotier pédonculé ce qui ne permet donc pas de relier la structure biochimique des TC à leur effet au niveau de l'intestin.

\section{d) Matières azotées excrétées et rete-} nues par l'animal

L'action des TC sur la digestion des matières azotées dans le rumen et dans l'intestin a des conséquences importantes sur la nature et la voie d'excrétion des matières azotées non utilisées et sur l'azote retenu par l'animal. La diminution du taux de $\mathrm{NH}_{3}$ dans le rumen en présence de TC conduit à une diminution de l'azote excrété dans l'urine (Scharenberg et al 2007). L'utilisation du PEG a permis de montrer que cette diminution de l'excrétion azotée dans l'urine est bien la conséquence de l'action des TC (Theodoridou et al 2010, 2012) et les résultats de Aufrère et al (2008) montrent que l'excrétion d'azote dans l'urine est nettement réduite avec le sainfoin par rapport à la luzerne (figure 7). En revanche, la baisse de digestibilité intestinale des matières azotées entraîne une augmentation de l'excrétion azotée dans les fèces comme le confirment les résultats obtenus avec du fourrage vert et de l'ensilage mi-fané de sainfoin (Theodoridou et al 2010, 2012), du sainfoin déshydraté ou du foin (Scharenberg et al 2007, 2008), de l'ensilage de ray-grass auquel il a été rajouté du mimosa (Deaville et al 2010). Une partition semblable de l'a- zote excrété a été trouvée par Waghorn et al (1994b), Mc Nabb et al (1996) avec du lotier pédonculé et par Bermingham et al (2001) avec du sulla, de même que pour des vaches laitières alimentées avec du ray-grass auquel il avait été rajouté des proportions importantes de lotier corniculé (Woodward et al 2009).

$\mathrm{Au}$ final, dans les comparaisons avec et sans PEG et entre sainfoin et luzerne la quantité totale d'azote excrétée n'est pas forcément modifiée, mais sa répartition entre urine et fèces l'est fortement (figure 7). Ces modifications sont susceptibles de limiter les pertes d'azote par lessivage et par volatilisation contribuant ainsi à réduire l'eutrophisation, l'impact sur la qualité de l'air et la production de gaz à effet de serre. En effet, il est connu que l'émission de $\mathrm{NH}_{3}$ dans l'air est défavorable à sa qualité. En particulier l'N urinaire est rapidement converti en $\mathrm{NH}_{3}$ puis en $\mathrm{NO}_{2}$ (Givens et Rulquin 2004, Kebread et al 2004). L'azote contenu dans les fèces serait moins polluant et contribuerait à l'accumulation de MO dans le sol alors que l'azote urinaire peut polluer l'eau du sol (Varel et al 1999, Grabber et al 2001).

En termes de nutrition pour le ruminant, bien que la digestibilité intestinale du sainfoin soit diminuée par rapport à celle de la luzerne (Aufrère et al 2008), la quantité d'azote retenu par l'animal n'est pas modifiée en présence de TC actifs (Theodoridou et al 2010). Les résultats de Theodoridou et al (2010, 2012) vont dans le même sens, que le sainfoin soit distribué en vert ou en ensilage mi-fané. Des résultats analogues ont été rapportés par Waghorn et Shelton (1997) pour du lotier pédonculé comparé à du ray-grass anglais. Scharenberg et al (2007) ont observé que les TC du sainfoin séché n'ont pas eu d'effet sur la rétention azotée alors qu'une diminution a été enregistrée avec du sainfoin déshydraté.

\section{6 / Quelles valeurs PDI et UF pour les légumineuses contenant des tannins condensés ?}

L'estimation de la teneur en PDI des fourrages nécessite de connaître : i) la teneur en MAT, ii) la dégradabilité théorique de matières azotées (DT), iii) la digestibilité réelle des protéines alimentaires dans l'intestin (dr), iv) la matière organique fermentescible, elle-même fonction de la matière organique digestible.

Les mesures de DT et de dr réalisées par les techniques en sachets de nylon pour du sainfoin et de la luzerne par Aufrère et al (2008) et par Theodoridou et al (2010) ont été comparées aux 
Tableau 2. Estimation de la valeur nutritive du sainfoin (valeurs PDI et UF) à partir des essais réalisés à I'INRA de Theix entre 2008 et 2010.

Comparaison avec les valeurs PDI estimées par les équations INRA 2007, et avec les valeurs UF figurant dans les tables INRA 2007 (valeurs dans les colonnes grisées).

\begin{tabular}{|c|c|c|c|c|c|c|c|c|c|c|c|c|c|c|}
\hline \multirow[b]{2}{*}{$\mathrm{g} / \mathrm{kg} \mathrm{MS}$} & \multicolumn{4}{|c|}{$\begin{array}{l}\text { Sainfoin }^{(1)} \text { variété Zeus } \\
\text { Fourrage vert }\end{array}$} & \multicolumn{6}{|c|}{$\begin{array}{c}\text { Sainfoin }{ }^{(2)} \text { variété Perly } \\
\text { Fourrage vert }\end{array}$} & \multicolumn{4}{|c|}{$\begin{array}{c}\text { Sainfoin }{ }^{(3)} \text { variété Perly } \\
\text { Enrubannage }\end{array}$} \\
\hline & Végétatif & & $\begin{array}{l}\text { Début } \\
\text { floraison }\end{array}$ & & $\begin{array}{c}\text { Fin } \\
\text { floraison }\end{array}$ & & $\begin{array}{l}\text { Fructi- } \\
\text { fication }\end{array}$ & & $\begin{array}{l}\text { Repousses } \\
\text { (5 sem.) }\end{array}$ & & $\begin{array}{c}\text { Fin } \\
\text { floraison }\end{array}$ & & $\begin{array}{l}\text { Repousses } \\
\text { (5 sem.) }\end{array}$ & \\
\hline MAT & 161 & & 124 & & 143 & & 145 & & 187 & & 140 & & 174 & \\
\hline DT $(\%)$ & 59,1 & 76,1 & 62.5 & 72,7 & 53,4 & 74,5 & 52,8 & 74,7 & 57,3 & 78,2 & 69,1 & 78,7 & 62,0 & 80 \\
\hline $\operatorname{dr}(\%)$ & 49,2 & 80,5 & 44,6 & 76,4 & 46,7 & 78.5 & 52,4 & 78,7 & 49,6 & 79,4 & 17,8 & 67,6 & 39,1 & 69,5 \\
\hline PDIA & 36 & 34 & 23 & 29 & 35 & 32 & 40 & 32 & 44 & 36 & 9 & 22 & 29 & 27 \\
\hline PDIMN & 51 & 68 & 42 & 50 & 40 & 59 & 40 & 60 & 57 & 82 & 53 & 62 & 58 & 78 \\
\hline PDIME & 50 & 52 & 51 & 52 & 42 & 45 & 36 & 39 & 44 & 47 & 35 & 39 & 41 & 46 \\
\hline PDIN & 87 & 102 & 65 & 79 & 74 & 91 & 80 & 92 & 101 & 118 & 62 & 84 & 87 & 105 \\
\hline PDIE & 86 & 86 & 74 & 80 & 77 & 76 & 76 & 71 & 88 & 83 & 44 & 61 & 69 & 73 \\
\hline $\mathrm{dMO}(\%)$ & 71,2 & & 68,2 & 71 & 61,0 & 62 & 56,0 & & 65,0 & & 54,0 & & 62,0 & \\
\hline $\begin{array}{l}\text { UFL ( } / \mathrm{kg} \\
\text { MS) }\end{array}$ & 0,90 & & 0,86 & 0,83 & 0,69 & 0,70 & 0,60 & & 0,74 & & 0,56 & & 0,70 & \\
\hline $\begin{array}{l}\text { UFV (/kg } \\
\text { MS) }\end{array}$ & 0,85 & & 0,80 & 0,77 & 0,60 & 0,61 & 0,50 & & 0,66 & & 0,46 & & 0,61 & \\
\hline
\end{tabular}

(1) Aufrère et al 2008 ; (2) Theodoridou et al 2010 ; (3) Theodoridou et al 2011a.

valeurs calculées à partir de la teneur en MAT selon l'équation proposée par Nozières et al (2007) et utilisée pour les calculs des Tables INRA 2007 (Baumont et al 2007). Pour le sainfoin, les valeurs de DT et de dr mesurées sont plus faibles que celles calculées (tableau 2). En revanche pour la luzerne, les valeurs de DT et de dr mesurées sont très cohérentes avec celles calculées à partir de la teneur en MAT. Cela est logique puisque la base de données utilisée pour les calculs des tables 2007 n'intégrait pas de mesures obtenues pour fourrages contenant des TC.

Au final, les valeurs PDIA estimées à partir des mesures sont proches de celles estimées à partir des équations utilisées pour les Tables INRA 2007 (tableau 2). En effet, la surestimation de la digestibilité intestinale compense la sous-estimation de la quantité de MAT échappant à la dégradation dans le rumen. De même, les valeurs PDIE estimées à partir des mesures sont proches de celles qui peuvent être estimées à partir des équations INRA 2007. En revanche, le calcul fait à partir des équations des tables INRA surestime la valeur PDIN du sainfoin de 10 à $20 \mathrm{~g}$ environ. Les estimations faites à partir des mesures expérimentales montrent que les valeurs PDIN et PDIE du sainfoin sont nettement plus équilibrées que celles de la luzerne.

Les données de dMO issues de ces mêmes expérimentations sur le sainfoin ont été utilisées pour le calcul de la valeur énergétique des fourrages contenant des TC (tableau 2). Les valeurs obtenues sont proches de celles des Tables INRA 2007 pour les stades de développement correspondants et du même ordre de grandeur que celles obtenues par Chassagne et Chambon (1993).

\section{7 / Utilisation dans la pratique des fourrages contenant des TC}

L'utilisation de ces fourrages demeure limitée en France. Ils ont fait l'objet de peu d'amélioration génétique. Souvent l'implantation de la culture est difficile. Par rapport à la luzerne, leur rendement peut être faible (cas du lotier corniculé), leur pérennité peut être limitée (cas du sainfoin). De plus, certains comme le sulla ne résistent pas au gel.

\section{a) Conservation en ensilage ou enru-} bannage

Ces fourrages utilisés traditionnellement en foin peuvent aussi être pâturés sans risque de météorisation. Une voie très prometteuse pour leur utilisation est la conservation en ensilage mi-fané moins dépendante du climat que la conservation en foin et permettant d'obtenir un fourrage de bonne qualité. La présence de TC capables de se fixer aux protéines entraîne une moindre hydrolyse de ces dernières dans le silo et une bonne conservation de l'ensilage de sainfoin sans ajout de conservateur (Theodoridou et al 2012). De plus, les teneurs élevées en glucides solubles permettent un développement des lactobacilles qui en transformant les glucides solubles en acide lactique entraîneront une baisse supplémentaire du $\mathrm{pH}$ et une bonne conservation de l'ensilage.

\section{b) Utilisation dans des mélanges d'espèces}

Les mélanges d'espèces associant des graminées et légumineuses permettent de fournir aux ruminants une alimentation de qualité, régulièrement produite au cours de l'année et d'éviter les risques de gaspillage d'azote tant au niveau de la culture que de l'utilisation par l'animal. Traditionnellement, le sainfoin est semé avec de la fétuque ou de la fléole mais il peut également être mélangé avec d'autres espèces.

L'introduction dans les mélanges de légumineuses contenant des TC est une approche astucieuse dans la mesure où la dégradabilité des protéines dans le rumen du mélange est inférieure à la moyenne de celles des fourrages initiaux, comme l'ont montré in vitro Julier et al (2002) pour un mélange luzerne lotier, Aufrère et al (2005) pour un mélange sainfoin luzerne, Niderkorn et al (2011) pour un mélange sainfoin dactyle, et in vivo (Aufrère et al non publié) pour un mélange sainfoin luzerne. Dans ce dernier cas, $75 \%$ de sainfoin serait nécessaire pour observer un effet sur la ration. Wang et al (2007) observent également un effet positif de mélanges sainfoin luzerne utilisés en ensilage ou en foin à la fois sur la qualité de l'ensilage et sur la digestibilité chez le mouton. Ces mélanges auraient un effet positif sur l'ingestibilité, la dégradation de l'azote chez le ruminant à condition que la quantité de TC soit suffisante. Ainsi Waghorn et Shelton (1997), n'observent pas d'interaction sur la valeur nutritive entre du ray-grass et du lotier corniculé contenant $1 \%$ de TC. L'effet de mélanges contenant des TC ou d'autres composés secondaires, distribués à l'auge en fourrage vert ou en ensilage sur la valeur alimentaire est en cours d'étude actuellement, en particulier dans le programme européen «Legume plus » qui a débuté en janvier 2012. 
Figure 8. Schéma récapitulatif des principaux effets potentiels des tannins condensés pour des fourrages utilisés dans des zones tempérées (d'après Patra et Saxena 2011).

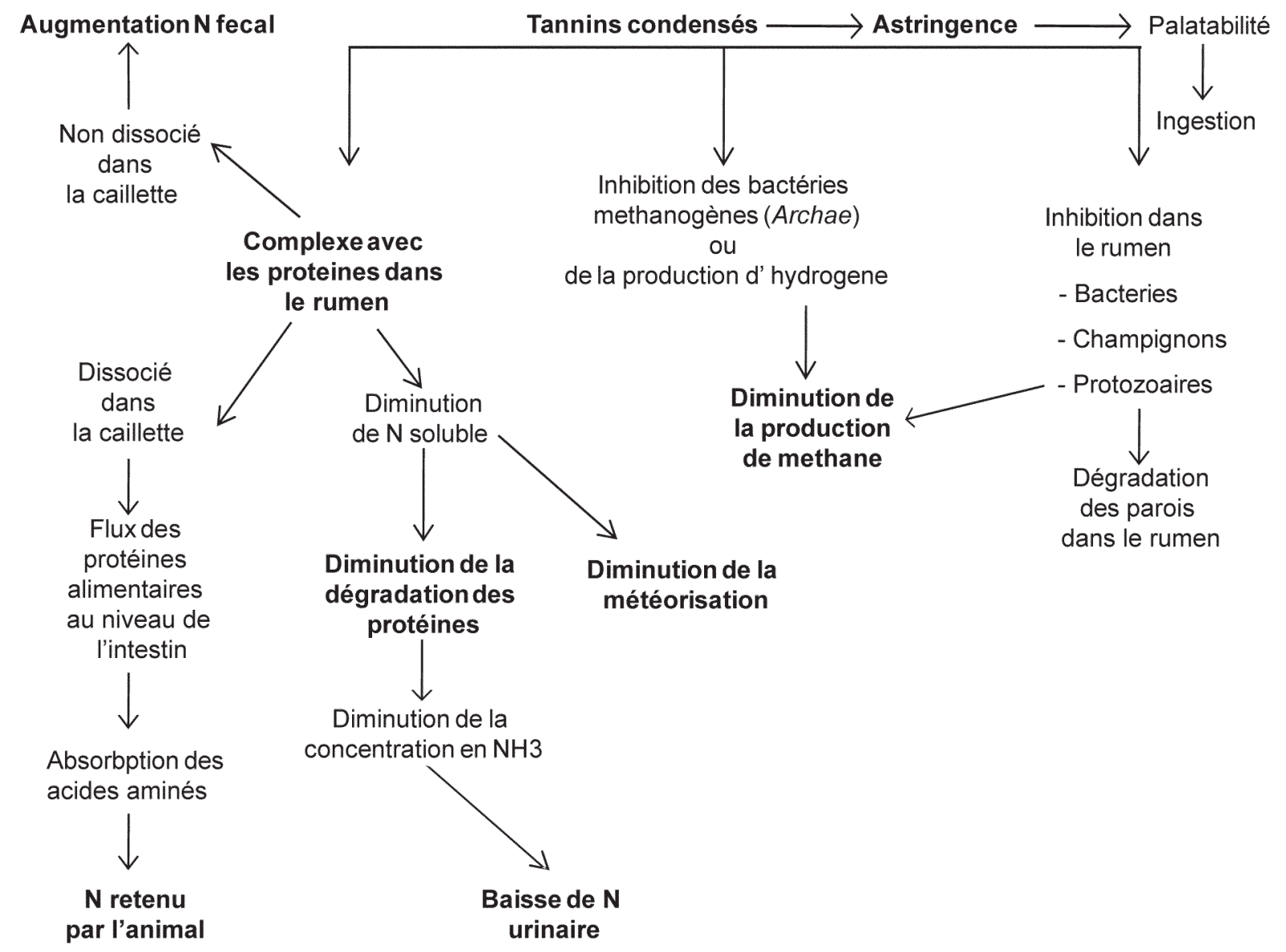

\section{Conclusion}

Les relations entre la teneur en TC des légumineuses, leur structure biochimique et leur activité biologique sont complexes. Elles varient selon l'espèce végétale, la variété, les conditions de culture, le stade de développement et le mode de conservation du fourrage. Il en résulte que les effets des TC sur les paramètres de la valeur alimentaire (ingestibilité, digestibilité de la matière organique, dégradation des matières azotées) ne sont pas univoques. Ces effets ne dépendent pas seulement de la teneur en TC dans le fourrage, mais aussi d'autres paramètres liés à la structure des TC. La détermination du poids moléculaire, du rapport $\mathrm{PD} / \mathrm{PC}$, de la conformation dans l'espace des TC, semble prometteuse pour expliquer leurs effets digestifs. Néanmoins, les questions liées aux méthodes de dosage ne sont pas entièrement résolues.

Les effets des TC sur la valeur alimentaire sont récapitulés dans la figure 8. Cette synthèse indique que la présence de TC en quantité modérée dans certaines légumineuses fourragères cultivées en zone tempérée, entraîne peu de modifications de la digestibilité par rapport à un fourrage témoin ne contenant pas de tannin. En revanche, pour la valeur azotée, la présence de TC entraîne généralement une diminution de la dégradation des protéines dans le rumen et une augmentation ou une diminution de la digestibilité dans l'intestin.
L'azote retenu par l'animal n'est pas ou peu modifié. Une diminution de l'azote excrété dans l'urine est généralement observée, compensée par une augmentation de l'azoté excrété dans les fèces, ce qui est favorable à la réduction des pertes d'azote par lessivage et volatilisation contribuant ainsi à réduire l'eutrophisation, et la production de gaz à effet de serre.

A partir des mesures expérimentales de digestibilité, de DT et de dr, il apparaît que les estimations proposées pour le sainfoin par les équations des tables INRA 2007 donnent des valeurs cohérentes pour la valeur énergétique, et la valeur PDIE. En revanche les valeurs PDIN sont surestimées et devraient être diminuées de 10 à $20 \mathrm{~g} / \mathrm{kg}$ de MS. 


\section{Références}

Aerts R., Barry T., McNabb W.C., 1999. Polyphenols and agriculture: beneficial effects of proanthocyanidins in forages. Agric. Ecosyst. Env., 75, 1-12.

Albrecht K.A., Muck R.E., 1991. Proteolysis in ensiled legumes that vary in tannin concentration. Crop Science, 31, 464-469.

Animut G., Puchala R., Goetsch A.L., Patra A.K., Sahlu T., Varel V.H., Wells J., 2008. Methane emission by goats consuming diets with different levels of condensed tannins from lespedeza. Anim. Feed Sci. Technol., 144, 212-227.

Anuraga M., Duarsa P., Hill M.J., Lovett J.V., 1993. Soil moisture and temperature affect condensed tannin concentrations and growth in Lotus corniculatus and Lotus pedunculatus. Aust. J. Agric. Res., 44, 1667-1681.

Arrigo Y., 2009. Effet d'un apport croissant d'esparcette dans des rations d'ensilage de luzerne sur la valeur nutritive des rations chez le mouton. Schriftenreihe aus dem Institut für Nutztierwissenschaften der ETH Zürich, 31, 120-123.

Arrigo Y., Scharenberg A., 2008. Digestibilité et dégradabilité de la matière azotée des plantes riches en tannins. Renc. Rech. Rum., 15, p281.

Arrigo Y., Dohme F., 2009. Influence de la distribution d'esparcette riche en tannins sur le métabolisme protéique des vaches au pâturage. Renc. Rech. Rum., 16, p75.

Aufrère J., Dudilieu M., Poncet C., Baumont R., 2005. Effect of condensed tannins in sainfoin on in vitro protein solubility of lucerne. Int. Grassland Congress, O'Mara F.P. (Ed).Wageningen Academic Publishers, p248.

Aufrère J., Baumont R., Delaby L., Pecatte J.R., Andrieu J., Andrieu J.P., Dulphy J.P., 2007. Prévision de la digestibilité des fourrages par la méthode pepsine-cellulase. Le point sur les équations proposées. In : Alimentation des ruminants. Agabriel J. (Ed). Dossier, INRA Prod. Anim., 20, 129-136.

Aufrère J., Dudilieu M., Poncet C., 2008. In vivo and in situ measurements of the digestive characteristics of sainfoin in comparison with lucerne fed to sheep as fresh forages at two growth stages and as hay. Animal, 2, 13311339

Barry T.N., Duncan S.J., 1984. The role of condensed tannins in the nutritional value of Lotus pedunculatus for sheep. 1. Voluntary intake. Brit. J. Nut., 51, 485-491.

Barry T.N., Forss D.A., 1983. The condensed tannin content of vegetative lotus pedunculatus, its regulation by fertiliser application and effect upon protein solubility. J. Sci. Food Agric., 34, 1047-1056.

Barry T.N., Manley T.R., 1984. The role of condensed tannins in the nutritional value of Lotus pedunculatus for sheep. 2. Quantitative digestion of carbohydrate and proteins. Brit. J. Nutr., 51, 493-504.

Barry T.N., Manley T.R., 1985. The role of condensed tannins in the nutritional value of Lotus pedunculatus for sheep. 3. Rates of body and wool growth. Brit. J. Nutr., 54, 211-217.

Barry T.N., Manley T.R., Duncan S.J., 1986. The role of condensed tannins in the nutritional value of Lotus pedunculatus for sheep. 4. Sites of carbohydrate and protein digestion as influenced by dietary reactive tannin concentration. Brit. J. Nutr., 55, 123-137.

Barry T.N., Manley T.R., 1986. Interrelationships between the concentrations of total condensed tannin, free condensed tannin and lignin in Lotus sp. and their possible consequences in ruminant nutrition. J. Sci. Food Agric., 37, 248-254.

Barry T.N., McNabb W.C., 1999. The implications of condensed tannins on the nutritive value of temperate forages fed to ruminants. A review article. Brit. J. Nutr., 81, 263-272.

Baumont R., Dulphy J.P., Sauvant D. Meschy F., Aufrère J., Peyraud J.L., 2007. Valeur alimentaire des fourrages et des matières premières : Tables et Prévision. In Alimentation des bovins, ovins et caprins. Tables INRA, 149-165.

Bhatta R., Uyeno Y., Tajima K., Takenaka A., YabumotoY., Nonaka I., Enishi O Kurihara M., 2009. Difference in the nature of tannins on in vitro ruminal methane and volatile fatty acid production and on methanogenic Archaea and protozoal populations. J. Dairy Sci., 92, 5512-5522.

Bell A.A., El-Zik K.M., Thaxton P.M., 1992. Chemistry, biological significance, and genetic control of proanthocyanidins in cotton (Gossypium spp.). In: Hemingway R.W., Laks P.E. (Eds). Plant polyphenols, synthesis, properties, significance. Plenum Press, London, UK, 571-595.

Bermingham E.N., Hutchinson K.J., Revel D.K., Brookes I.M., McNabb W.C., 2001. The effect of condensed tannins in sainfoin (Onobrycis viciifolia) and sulla (Hedysarum coronarium) on the digestion of amino acids in sheep. Proc. N.Z. Soc. Anim., 61, 116-119.

Borreani G., Peiretti P.G., Tabacco E., 2003. Evolution of yield and quality of sainfoin (Onobrychis viciifolia Scop.) in the spring growth cycle. Agronomie, 23, 193-201.

Broadhurst R.B., Jones W.T., 1978. Analysis of condensed tannins using acidified vanillin. J. Sci. Food Agric., 29, 788-794

Chassagne J., Chambon J., 1993. Sainfoin: legume adapted to the soil of causses. application to the agricultural region of Gramat and to that of Limoges (France). Journées d'information de l'AFPF (Association Française pour la Production Fourragère). Fourrages, 134, 177 181

Cheynier V., 2005. Polyphenols in foods are more complex than often thought. Am. J. Clin. Nutr., 81, 223S-229S

Chiquette J., Cheng K.J., Costerton J.W. Milligan L.P., 1988. Effect of tannins on the digestibility of two isosynthetic strains of birdsfoot trefoil (Lotus corniculatus L.) using in vitro and in sacco techniques. Can. J. Anim. Sci., 68, 751-760.

Deaville E.R., Givens D.I., Mueller-Harvey I., 2010. Chesnut and mimosa tannin silages: Effects in sheep differ for apparent digestibility, nitrogen utilisation and losses. Anim. Feed Sci. Technol., 157, 129-138.

Douglas G.B., Wang Y., Waghorn G.C. Barry T.N., Purchas RW., Foote A.G., Wilson G.E., 1995. Liveweight gain and wool production of sheep grazing Lotus corniculatus and Lucerne (Medicago sativa). New Zeal. J. Agric. Res., 38, 95-104.
Douglas G.B., Stienezen M., Waghorn G.C., Foote A.G., 1999. Effect of condensed tannins in birdsfoot trefoil (Lotus corniculatus) and sulla (Hedysarum coronarium) on body weight, carcass fat depth, and wool growth of lambs in New Zealand. N.Z. J. Agric. Res., 42, 55-64.

Egan A.R., Ulyatt M.J., 1980. Quantitative digestion of fresh herbage by sheep. J. Agric. Sci., 94, 47-56.

Folin O., Denis W., 1912. Tyrosine in proteins as determined by a new colorimetric method. J. Biol. Chem., 245-251.

Foo L.Y., Lu Y., McNabb W.C., Waghorn G.C., Ulyatt M.J., 1997. Proanthocyanidins from Lotus pedunculatus. Phytochemistry, 45, 1689-1696

Fraser M.D., Fychan R., Jones R., 2000. Voluntary intake, digestibility and nitrogen utilization by sheep fed ensiled forage legumes. Grass Forage Sci., 55, 271-279.

Frazier R.A., Deaville E.R., Green R.J., Willoughby I., Stringano E., Plan J., MuellerHarvey I., 2010. Interactions of tea tannins and condensed tannins with proteins. J. Pharm. Biomed. Anal., 51, 490-495.

Frutos P., Hervas G., Ramos G., Giraldez F.J., Mantecon A.R., 2002.Condensed tannin content of several shrub species from a mountain area in northern Spain, and its relationship to various indicators of nutritive value. Anim. Feed Sci. Technol., 95,215-226.

Frutos P., Hervas G., Giraldez F.J., Mantecon A.R., 2004. Review, tannins and ruminant nutrition. Span. J. Agric. Res., 2, 191-202.

Gea A., Stringano E., Brown R.H., MuellerHarvey I., 2011. In situ analysis and structural elucidation of sainfoin (Onobrychis viciifolia) tannins for high throughput germplasm screening. J. Agric. Food Chem., 59, 495-503.

Gebrehiwot L., Beuselink P.R., Roberts C.A., 2002. Seasonal variations in condensed tannin concentration of three Lotus species. Agron. J., 94, 1059-1065.

Givens D.I., Rulquin H., 2004. Utilization by ruminants of nitrogen compounds in silagebased diets. Anim. Feed Sci. Technol., 114, 1-18.

Goldstein J.L., Swain T., 1965. The inhibition of enzymes by tannins. Phytochemistry, 4 185-192.

Grabber J., Broderick G., Russelle M. Powell M, Rotz A. Muck R., Mertens D. Maasingill L., 2001. Tannin containing forage crops: A way to improve nitrogen use and profitability of dairy farms? U.S.Dairy Forage Research Center, 20th anniversary, 29-30 June 2001

Guglielmelli A., Calabro S., Primi R., Carone F., Cutrignelli M.I., Tudisco R., Piccolo G., Ronchi B., Danieli P.P., 2011. In vitro fermentation patterns and methane production of sainfoin (Onobrychis Viciifolia Scop.) hay with different condensed tannin contents. Grass Forage Sci., 66, 488-500.

Hagerman A.E., 1987. Radial diffusion method for determining tannin in plant extracts. J. Chem. Ecol., 13, 437-449.

Harrison D.G., Beever D.E., Thomson D.J., Osbourn D.F., 1973. The influence of diet upon the quantity and types of amino acids entering 
and leaving the small intestine of sheep. J Agric. Sci. Camb., 81, 391-401.

Hedqvist H., Mueller-Harvey I., Reed J.D., Krueger C.G., Murphy M., 2000. Characterisation of tannins and in vitro protein digestibility of several Lotus corniculatus varieties. Anim. Feed Sci. Technol., 87, 41-56.

Iason G.R., Hartley S.E., Duncan A.J., 1993. Chemical composition of Calluna vulgaris (Ericaceae): do responses to fertilizer vary with phenological stage. Biochem. Syst. Ecol., $21,315-321$

Iason G.R., Hodgson J., Barry T., 1995. Variation in condensed tannin concentration of a temperate grass (Holcus Lanatus) in relation to season and reproductive development. J. Chem. Ecol., 21, 1103-1112.

INRA, 2007. Alimentation des bovins, ovins et caprins. Besoins des animaux. Valeurs des aliments. Tables INRA 2007. Editions Quae, Paris, France, 307p.

Jayanegara A., Togtokhbayar N., Makkar H.P.S., Becker K., 2009. Tannins determined by various methods as predictors of methane production reduction potential of plants by an in vitro rumen fermentation system. Anim. Feed Sci. Technol., 150, 230-237.

Jones W.T., Mangan J.L., 1977. Complexes of the condensed tannins of sainfoin (Onobrychis viciifolia) with fraction 1 leaf protein and with submaxillary mycoprotein, and their reversal by polyethylene glycol and $\mathrm{pH}$. J. Sci. Food Agric., 28, 126-136.

Julier B., Lila M., Huygue C., Morris P., Allison G., Robins M., 2002. Effects of condensed tannin content on protein solubility in legume forages. In: Multi-function grasslands: quality forages, animal products and landscapes. Proc. $19^{\text {th }}$ Gen. Meet. Eur. Grassland Fed., $27-$ 30 May, La Rochelle, France, 134-135.

Kalu B.A., Fick G.W., 1981. Quantifying morphological development of alfalfa for studies of herbage quality. Crop Sci., 21, 267-271.

Karabulut A., Canbolat O., Kamalak A., 2006. The effect of PEG on in vitro organic matter digestibility and metabolizable energy of Lotus corniculatus L. Lotus Newsletter, 36, 7-10.

Kebreab E., Mills J.A.N., Crompton L.A., Bannik A., Dijkstra J., Gerrits W.J.J., France J., 2004. An integrated mathematic model to evaluate nutrient partition in dairy cattle between animal and environment. Anim. Feed Sci. Technol., 112, 131-154.

Kelman W.M., Tanner G.J., 1990. Foliar condensed tannin levels in lotus species growing on limed and unlimed soils in South- Eastern Australia. Proc. N.Z. Grassland. Assoc., 52, 51-54.

Koupai-Abyazani M.R., McCallum J., Muir A.D., Bohm B.A., Towers G.H.N., Gruber M.Y., 1993. Developmental changes in the composition of proanthocyanidins from leaves of sainfoin (Onobrychis viciifolia Scop.) as determined by HPLC analysis. J. Agric. Food Chem., 41, 1066-1070.

Kraiem K., Garrett J.E., Meiske J.C., Goodrich R.D., Marten G.C., 1990. Influence of method of forage preservation on fibre and protein digestion in cattle given lucerne, birdsfoot trefoil and sainfoin. Anim Prod., 50, 221230 .

Lascano C.E., Schmidt A., Barahona R., 2001. Forage quality and environement. In:
Intern. Grassland Cong., 19, São Pedro. Proc. São Pedro: FEALQ. 351-356.

Lees G.L., Hinks C.F., Suttill N.H., 1994 Effect of high temperature on condensed tannin accumulation in leaf tissues of big trefoil (Lotus uliginosus Schkur). J. Sci. Food Agric. $65,415-421$.

Lees G.L., Gruber M.Y., Suttill N.H., 1995. Condensed tannnins in sainfoin II. Occurrence and changes during leaf development. Can. J. Bot., 73, 1540-1547.

Makkar H.P.S., Blummel M., Becker K 1995. Formation of complexes between polyvinyl pyrrolidones or polyethylene glycols and tannins, and their implication in gas production and true digestibility in in vitro techniques. Brit. J. Nutr., 73, 897-913.

Mangan J.L., 1988. Nutritional effects of tannins in animal feeds. Nutr. Res. Rev., 1, 209-231.

Marais J.P.J., Mueller-Harvey I., Brandt E.V., Ferreira D., 2000. Polyphenols, condensed tannins, and other natural products in Onobrychis viciifolia (sainfoin). J. Agric. Food Chem., 48, 3440-3447.

McAllister T.A., Martinez T., Bae H.D. Muir A.D., Yanke L.J., Gones G.A., 2005. Characterization of condensed tannins purified from legume forages: Chromophore production, protein precipitation and inhibitory effects on cellulose digestion. J. Chem. Ecol., 31, 2049-2068

McMahon L.R., McAllister T.A., Berg B.P., Majak W., Acharya S.N., Popp J.D., 2000. A review of the effects of forage condensed tannins on ruminal fermentation and bloat in grazing cattle. Can. J. Plant Sci., 80, 469-485.

McNabb W.C., Waghorn G.C., Peters J.S. Barry T.N., 1996. The effect of condensed tannins in Lotus pedunculatus on the solubilization and degradation of ribulose-1, 5-bisphosphate carboxylase (EC 4.1.1.39; Rubisco) protein in the rumen and the sites of Rubisco digestion. Brit. J. Nutr., 76, 535-549.

Meagher L.P., Lane G., Sivakumaran S. Tavendale M.H., Fraser K., 2004. Characterization of condensed tannins from Lotus species by thiolytic degradation and electrospray mass spectrometry. Anim. Feed Sci. Technol., 117, 151-163.

Min B.R., Fernandez J.M., Barry T.N. McNabb W.C., Kemp P.D., 2001. The effects of CT in Lotus corniculatus upon reproductive efficiency and wool production in ewes during autumn. Anim. Feed Sci. Technol., 92, 185-202.

Min B.R., Barry T.N., Attwood G.T. McNabb W.C., 2003. The effect of condensed tannins on the nutrition and health of ruminants fed fresh temperate forages: a review. Anim. Feed Sci. Technol., 106, 3-19.

Minneé E.M.K., Woodward S.L., Waghorn G.C., Laboyrie P.G., 2002. The effect of ensiling forage legumes on condensed tannins. Agron. N.Z. 32/33, 117-119.

Molan A.L., Attwood G.T., Min B.R., McNabb W.C., 2001. The effect of condensed tannin from Lotus corniculatus and Lotus pedunculatus on the growth of proteolytic rumen bacteria in vitro and their possible mode of action. Can. J. Microbiol., 47, 626-633.

Mole S., Waterman P.G., 1987. Tannic acid and proteolytic enzymes : enzyme inhibition or substrate deprivation. Phytochemistry, 26, 99102.
Molle G., Decandia M., Giovanetti V., Cabiddu A., Fois N., Sitzia M., 2009. Responses to condensed tannins of flowering sulla (Hedysarum coronarium L.) grazed by dairy sheep. Part 1: Effects on feeding behaviour, intake, diet digestibility and performance. Livestock Sci., 123, 138-146.

Mueller-Harvey I., 2006. Unravelling the conundrum of tannins in animal nutrition and health. J. Sci. Food Agric., 86, 2010-2037.

Niderkorn V., Baumont R., Le Morvan A., Macheboeuf D., 2011. Occurrence of associative effects between grasses and legumes in binary mixtures on in vitro rumen fermentation characteristics. J. Anim. Sci., 89, 1138-1145.

Nozières M.O., Dulphy J.P., Peyraud J.L., Poncet C., Baumont R., 2007. La valeur azotée des fourrages. Nouvelles estimations de la dégradabilité des protéines dans le rumen et de la digestibilité réelle des protéines alimentaires dans l'intestin grêle: conséquences sur les valeurs PDI. In : Alimentation des Ruminants. Agabriel J. (Ed). Dossier, INRA Prod. Anim. 20, 109-118.

Pagan S., Wolfe R.M., Terrill T.H., Muir J.P., 2009. Effect of drying method and assay methodology on detergent fiber analysis in plants containing condensed tannins. Anim. Feed Sci. Technol., 154, 119-124.

Parker R.J., Moss B.R., 1981. Nutritional value of sainfoin hay compared with alfalfa hay. J Dairy Sci., 64, 206-210.

Patra K., Saxena J., 2011. Exploitation of dietary tannins to improve rumen metabolism and ruminal nutrition . J. Sci. Food Agric., 91, 24-37.

Pilluzza G., Bullita S., Deroma M., Odoard M., 2000. The accumulation of condensed tannins in local populations of sulla. Cahiers Options Méditerranéennes, 45, 199-202.

Porter L.J., Woodruffe J., 1984. Haemanolysis: the relative astringency of proanthocyanidin polymers. Phytochemistry, 32, 447-453.

Porter L.J., Hrstich L.N., Chan B.G., 1986. The conversion of procyanidins and prodelphinidins to cyanidin and delphinidin. Phytochemistry, 1, 223-230.

Ramírez-Restrepo C.A., Barry T.N., LopezVillalobos N., 2006. Organic matter digestibility of condensed tannin-containing Lotus corniculatus and its prediction in vitro using cellulase/hemicellulase enzymes. Anim. Feed Sci. Technol., 125, 61-71.

Reed J.D., Horvath P.J., Allen M.S., Van Soest P.J., 1985. Gravimetric determination of soluble phenolics including tannins from leaves by precipitation with trivalent ytterbium. J. Sci. Food Agric., 33, 213-220

Sarni-Manchado P., Cheynier V., Moutoune M., 1999. Interactions of grape seed tannins with salivary proteins. J. Agric. Food Chem., 47, 42-47.

Scharenberg A., Arrigo Y., Gutzwiller A. Wyss U., Hess H., Kreuzer M., Dohme F. 2007. Effect of feeding dehydrated and ensiled tanniferous sainfoin (Onobrychis viciifolia) on nitrogen and mineral digestion and metabolism of lambs. Arch. Anim. Nutr., 61, 390-405.

Scharenberg A., Heckendorn F., Arrigo Y. Hertzberg H., Gutzwiller A., Hess H.D., Kreuzer M., Dohme F., 2008. Nitrogen and mineral balance of lambs artificially infected with Haemonchus contortus and fed tanni- 
ferous sainfoin (Onobrychis viciifolia). J. Anim. Sci., 86, 1879-1890.

Schiller L.R., Emmett M., Santa Ana C.A., Fordtran J.S., 1988. Osmotic effects of polyethylene glycol. Gastroenterology, 94, 933941 .

Schofield P., Mbugua D.M., Pell A.N., 2001. Analysis of condensed tannins: a review. Anim. Feed Sci. Technol., 91, 21-40.

Silber M.L., Davitt B., Khairutdinov R.F., Hurst J.K., 1998. A mathematical model describing tannin-protein association. Anal. Biochem., 263, 46-50.

Sivakumaran S., Meagher L.P., Foo L.Y. Lane G.A., Fraser K., Rumball W., 2004. Floral procyanidins of the forage legume red clover (Trifolium pratense L.). J. Agric. Food Chem., 52, 1581-1585.

Spencer C.M., Cai Y., Martin R., Gaffney S.H., Goulding P.N., Magnolato D., Lilley T.H., Haslam E., 1988. Polyphenol complexation-some thoughts and observations. Phytochemistry, 27, 2397-2409.

Stienezen M., Waghorn G.C., Douglas B. 1996. Digestibility and effects of condensed tannins on digestion of sulla (Hedysarum coronarium) when fed to sheep. N.Z. J. Agric. Res., 39, 215-221.

Tables méditerranéennes 1990. Tables of nutritive value for ruminants of mediterranean forages and by products. Options méditerranéennes, pp 42.

Tamminga S., Bannink A., Dijkstra J., Zom R., 2007. Feeding strategies to reduce methane loss in cattle. Report 34, Animal Science Group. http://edepot.wur.nl/28209.

Tavendale M.H., Meagher L.P., Pacheco D., Walker N., Attwood G.T., Sivakumaran S., 2005. Methane production from in vitro rumen incubations with Lotus pedunculatus and Medicago sativa and effects of extractable condensed tannin fractions on methanogenesis. Anim. Feed Sci. Technol., 123, 403-419.

Terrill T.H., Rowan A.M., Douglas G.B. Barry T.N., 1992a. Determination of extractable and bound condensed tannin concentrations in forage plants, protein concentrate meals and cereal grains. J. Sci. Food Agric., 58, 321-329.

Terrill T.H., Douglas G.B., Foote A.G., Purchas P.W., Wilson G.F., Barry T.N., 1992b. Effect of condensed tannins upon body growth, wool growth and rumen metabolism in sheep grazing sulla (Hedysarum coronarium) and perennial pasture. J. Agric. Sci. Cambridge, 119, 265-273.

Theodoridou K., Aufrère J., Andueza D., Pourrat J., Lemorvan A., Stringano E., Mueller-Harvey I., Baumont R., 2010. The effect of condensed tannins in fresh sainfoin (Onobrychis viciifolia) on in vivo and in situ digestion in sheep. Anim. Feed Sci. Technol., 160, 23-28.
Theodoridou K., 2010. Les effets des tannins condensés du sainfoin (Onobrychis viciifolia) sur digestion et sa valeur nutritive. Thèse Université Blaise Pascal, Clermont-Ferrand, France, 285p

Theodoridou K., Aufrère J., Andueza D., Lemorvan A., Picard F., Stringano E., Stringano E., Pourrat J., Mueller-Harvey I., Baumont R., 2011a. Effect of plant development during first and second growth cycle on chemical composition, condensed tannins and nutritive value of three sainfoin (Onobrychis viciifolia) varieties and Lucerne. Grass Forage Sci., 66, 402-414.

Theodoridou K., Aufrère J., Andueza D. Lemorvan A., Picard F., Pourrat J., Baumon R., 2012. Effect of condensed tannins in wrapped silage bales of sainfoin (Onobrychis viciifolia) on in vivo and in situ digestion in sheep. Animal, 6, 245-253.

Theodoridou K., Aufrère J., Niderkorn V., Andueza D., Lemorvan A., Picard F., Baumon R., 2011b. In vitro study of the effects of condensed tannins in sainfoin on the digestive process in the rumen at two vegetation cycles. Anim. Feed Sci. Technol., 170, 147-159.

Tibe O., Meagher L.P., Fraser K., Harding D.R.K., 2011. Condensed tannins of flavonoids from the forage legume sulla (Hedysarum coronarium). J. Agric. Food Chem., 59, 9402-9409.

Turgut L., Yanar M., 2004. In situ dry matter and crude protein degradation kinetics of some forages in Eastern Turkey. Small Ruminant Res., 52, 217-222.

Ulyatt M.J., Egan A.R., 1979. Quantitative digestion of fresh herbage by sheep: V. The digestion of four herbages and prediction of sites of digestion. J. Agric. Sci., 92, 605-616.

Van Soest P.J., Robertson J.B., 1980 Systems of analysis for evaluating fibrous feeds. In: standardization of analytical methodology for feeds. Pidgen W.J., Balch C.C Graham M., (Eds). Int. Dev. Res. Centre, Ottawa, Canada, 49-60.

Varel V.H., Nienaber J.A., Freetly H.C., 1999. Conservation of nitrogen in cattle feedlo waste with urease inhibitors. J. Anim. Sci., 77, 1162-1168.

Waghorn G.C., Ulyatt M.J., John A., Fisher M., 1987. The effect of condensed tannins on the site of digestion of amino acids and other nutrients in sheep fed on Lotus corniculatus $L$. Brit. J. Nutr., 57, 115-126.

Waghorn G.C., Shelton I.D., McNabb W.C., 1994a. Effects of condensed tannins in Lotus pedunculatus on its nutritive value for sheep. 1 Non-nitrogenous aspects. J. Agric. Sci., 123, 99-107.

Waghorn G.C., Shelton I.D., McNabb W.C., McCutcheon S.N., 1994b. Effects of condensed tannins in Lotus pedunculatus on its nutritive value for sheep. 2. Nitrogenous aspects. J. Agric. Sci., 123, 109-119.
Waghorn G.C., Shelton I.D., 1995. Effect of condensed tannins in Lotus pedunculatus on the nutritive value of ryegrass (Lolium perenne) fed to sheep. J. Agric. Sci., 125, 291297.

Waghorn G.C., Shelton I.D., 1997. Effect of condensed tannins in Lotus corniculatus on the nutritive value of pasture for sheep. J. Agric. Sci., 128, 365-372.

Waghorn G.C., Reed J.D., Ndlovu L.R., 1999. Condensed tannins and herbivore nutrition. In: Proc. $18^{\text {th }}$ Int. Grassland Cong., Vol. III, Buchanan-Smith J.G., Bailey L.D., McCaughy P. (Eds). Saskatchewan Canada, 8 19 June 1997. Association Management Centre, Calgary, AB, Canada, 153-166.

Waghorn G.C., Tavendale M.H., Woodfield D.R., 2002. Methanogenesis from forages fed to sheep. Proc. N.Z. Grassland Assoc., 64, 167171.

Waghorn G.C., 2008. Beneficial and detrimental effects of dietary condensed tannins for sustainable sheep and goat production. Progress and challenges. Anim. Feed Sci Technol., 147, 116-139.

Wang Y., Waghorn G.C., Barry T.N., Shelton I.D., 1994. The effect of condensed tannins in Lotus corniculatus on plasma metabolism of methionine, cysteine and inorganic sulphate by sheep. Brit. J. Nutr., 72, 923-935.

Wang Y., Barbieri L.R., Berg B.P., McAllister T.A., 2007. Effect of mixing sainfoin with alfalfa on ensiling, ruminal fermentation and total tract digestion of silage. Anim. Feed Sci. Technol., 135, 296-314.

Wang Y., Douglas G.B., Waghorn G.C., Barry T.N., Foote A.G., Purchas R.W., 1996. Effect of condensed tannins upon the performance of lambs grazing Lotus corniculatus and lucerne (Medicago sativa). J. Agric. Sci., 126 , 87-98.

Woodward S.L., Waghorn G.C., Lassey K.R., Laboyrie P.G., 2002. Does feeding sulla (Hedys coronarium) reduce methane emissions from dairy cows? Proc. N.Z. Anim. Prod., 62, 227-230

Woodward S.L., Waghorn G.C., Laboyrie P.G., 2004. Condensed tannins in birdsfoot trefoil (Lotus corniculatus) reduce methane emissions from dairy cows. Proc. N.Z. Anim. Prod., $64,160-164$

Woodward S.L., Waghorn G.C., Watkins K.A., Bryant M.A., 2009. Feeding birdsfoot trefoil (Lotus corniculatus) reduces the environmental impacts of dairy farming. Proc. N.Z. Anim. Prod., 69, 179-183. 


\title{
Résumé
}

Cette synthèse fait le point de l'état des connaissances sur la teneur en tannins condensés des légumineuses fourragères de milieu tempéré, leur structure et leur activité biologique, ainsi que sur les facteurs qui font varier ces paramètres. Les effets des tannins condensés sur l'ingestibilité, la digestion dans le rumen et dans l'intestin de la matière organique et des matières azotées sont passés en revue. A l'exception du lotier pédonculé, les tannins condensés affectent peu l'ingestibilité et la digestion de la matière organique. A l'inverse, la digestibilité des matières azotées est toujours diminuée en présence de tannins condensés. La baisse de la dégradabilité des matières azotées dans le rumen n'est que rarement compensée par une meilleure utilisation des acides aminés au niveau de l'intestin. Toutefois, la quantité d'azote retenu par l'animal n'est généralement pas diminuée ce qui est positif pour le ruminant sur le plan nutritionnel, et la part de l'azoté excrété dans l'urine est généralement réduite au profit de l'azoté excrété dans les fèces. La diminution du méthane éructé et de l'azote excrété dans l'urine, observée pour ces fourrages est favorable à la réduction des gaz à effet de serre et à la limitation des pertes d'azote par lessivage et volatilisation. Au final, les valeurs énergétiques et PDIE estimées à partir de mesures récentes sont cohérentes avec les valeurs des Tables INRA 2007. En revanche, les valeurs PDIN devraient être diminuées de 10 à $20 \mathrm{~g} / \mathrm{kg}$ MS par rapport aux estimations fournies par les équations INRA 2007.

\begin{abstract}
Feed value for ruminants of legume forages containing condensed tannins in temperate environment.

This review focuses on the state of knowledge of the content, biological activity, and structure of condensed tannins in tannin-rich legume forage species from temperate areas as well as the factors that influence these parameters. The effects of condensed tannins on feed intake, digestibility of organic matter and crude protein in the rumen and the intestine of ruminants are reviewed. With the exception of big trefoil, condensed tannins little affect intake and digestion of organic matter. In contrast, the digestibility of crude protein is always reduced in the presence of condensed tannins. The decrease in crude protein degradability in the rumen is rarely matched by better utilization of amino acids in the intestine. However, the amount of nitrogen retained by the animal is not diminished which is positive for ruminant nutrition, and the part of nitrogen excreted in urine is generally reduced. The decrease in methane eructed and urinary nitrogen excreted observed for these forages are in favor of reducing greenhouse gas emissions and limiting losses through leaching and volatilization. Finally, the energy value and the PDIE values estimated from recent measurements are consistent with the references provided in the INRA 2007 Tables. In contrast, the PDIN values should be reduced by 10 to $20 \mathrm{~g} / \mathrm{kg}$ DM compared to the values of INRA 2007 Tables.
\end{abstract}

AUFRERE J., THEODORIDOU K., BAUMONT R., 2012. Valeur alimentaire pour les ruminants des légumineuses contenant des tannins condensés en milieux tempérés. INRA Prod. Anim. 25, 29-44. 